\title{
Quantum Spin Chains with Quantum Group Symmetry
}

\author{
M. Fannes 1, 2, B. Nachtergaele ${ }^{3,4}$, R.F. Werner ${ }^{5}$ \\ ${ }^{1}$ Inst. Theor. Fysica, Universiteit Leuven, B-3001 Heverlee, Belgium \\ e-mail: mark.fannes@fys.kuleuven.ac.be \\ ${ }^{2}$ Onderzoeksleider, N.F.W.O. Belgium \\ ${ }^{3}$ Dept. of Physics, Princeton University, Princeton, NJ 08544-0708, USA \\ e-mail: bxn@math.princeton.edu \\ ${ }^{4}$ Supported in part by NSF Grant \# PHY90-19433 A02 \\ ${ }^{5}$ Fachbereich Physik, Universität Osnabrück, D-49069 Osnabrück, Germany \\ e-mail: reinwer@dosuni1.rz.uni-osnabrueck.de
}

Received: 6 June 1994/in revised form: 1 March 1995

\begin{abstract}
We consider actions of quantum groups on lattice spin systems. We show that if an action of a quantum group respects the local structure of a lattice system, it has to be an ordinary group. Even allowing weakly delocalized (quasi-local) tails of the action, we find that there are no actions of a properly quantum group commuting with lattice translations. The non-locality arises from the ordering of factors in the quantum group $C^{*}$-algebra, and can be made one-sided, thus allowing semi-local actions on a half chain. Under such actions, localized quantum group invariant elements remain localized. Hence the notion of interactions invariant under the quantum group and also under translations, recently studied by many authors, makes sense even though there is no global action of the quantum group. We consider a class of such quantum group invariant interactions with the property that there is a unique translation invariant ground state. Under weak locality assumptions, its GNS representation carries no unitary representation of the quantum group.
\end{abstract}

\section{Introduction}

Symmetry has always played an important role in theoretical physics in helping to reduce a problem with many variables to a more tractable size. In statistical mechanics we have infinitely many degrees of freedom to deal with, so often the symmetry, while helpful, is not sufficient to solve the problem, unless we have "infinitely many symmetries." One example is the theory of mean-field lattice systems, where the inherent permutation symmetry is sufficient to reduce the computation of the limit free energy density, of the possible limit states [FSV, RW], and of the limit dynamics [DW] to corresponding problems in the algebra for a single spin.

Another example, which has been studied intensively by many authors recently [Bab, BMNR, DC, KS, MN, GS], is the class of models which can be solved exactly (though not always rigorously) by means of the Bethe Ansatz. The basis of this method is to diagonalize the Hamiltonian along with an infinite set of constants of motion. In some cases the occurrence of this infinite set of constants of motion

Copyright (C) 1995 by the authors. Faithful reproduction of this article by any means is permitted for non-commercial purposes. 
is related to the appearance of a new kind of symmetry, called quantum group symmetry. This nourishes the hope that by relaxing the demands usually made on the structure of a symmetry group, and allowing the wider class of quantum groups, one can benefit from symmetry considerations in new situations, where a symmetry in the traditional sense is simply not present. A particularly interesting development in this direction is the integrable Haldane-Shastri model [Hal, Sha]. This quantum spin chain with long-range interactions can be interpreted as an ideal semion gas, i.e. the spinon excitations obey fractional statistics. For more details and generalizations we refer the reader to [Ha2] and the references therein. Another example where a quantum group symmetry plays an explicit role is in the study of non-translation invariant ground states of the ferromagnetic XXZ chain [GW, ASW].

In this paper we exclusively consider one-dimensional quantum spin systems, or "spin chains," as a testing ground for applications of quantum group symmetries. We emphasize that here we use the word "spin chain" in its meaning familiar from statistical mechanics, i.e. spins at different sites commute, and we do not consider modified (braided) tensor products [Maj]. A number of models of this type have been considered in the recent literature [KSZ, BY]. The interactions in these models are both translation invariant, like the usual lattice interactions, and quantum group invariant in a sense we will make more precise below. It is thus natural to ask for the quantum group symmetry of the relevant states - temperature and ground states - of these models. For example, could there be "spontaneous quantum symmetry breaking"? In the case of ordinary groups it is clear how to define such notions: the symmetry is implemented locally by unitaries, in a way which is compatible with the thermodynamic limit. The symmetry group thus acts by automorphisms on the infinite system described by the quasi-local algebra, and it is with respect to this action that we can talk about "invariant states" of the infinite system.

As we will show in this paper, however, this approach does not work for quantum group symmetries. The first limitation is that the formation of tensor products with non-abelian coefficients requires an ordering of the factors in the product, which is correlated with the algebraic ordering of factors in the algebra. This limits all considerations to one dimensional systems, or, in the field theoretical context, to systems with one space dimension and one time dimension. If we make the technical simplifications of choosing a discrete space variable, and a finite dimensional one-site algebra, we arrive at the setting of quantum spin chains, used in this paper. Given a unitary action on the one-site algebra, we can define a product action of a quantum group, for each finite segment of the chain. The fundamental difficulty, however, is that these actions are not compatible with the identifications used to form the inductive limit to the infinite system. More precisely, the compatibility holds for enlargement of the system towards the right, but not towards the left (or, conversely, depending on conventions). This means that we can define quantum group actions on a semi-infinite chain, but not on the full chain. We prove that this restriction is inherent in the quantum group concept, by showing that there is no action on the quasi-local algebra of the chain which commutes with translations.

In [DFJMN] Davies et al. study the quantum group symmetries of the antiferroelectric XXZ chain. The infinite dimensional symmetry algebra introduced there contains the finite dimensional quantum group $\mathrm{S}_{\nu} \mathrm{U}(2)$. Therefore our results, in particular Theorem 11, imply that the construction of [DFJMN] cannot lead to a proper action of the quantum symmetries on the observable algebra which commutes with the translations of the chain (or any infinite subgroup of the translations). It is an interesting open question in what sense such an action could be defined. 
In contrast to the actions defined for each segment, the sets of invariant elements for these actions are compatible with the inductive limit. This allows us to define quantum group invariant interactions (see, e.g. [MMP]), even though this invariance cannot be understood as invariance with respect to a global action.

The fact that the invariant elements have much better localization properties than general elements is reminiscent of the theory of superselection sectors in relativistic quantum field theory. Interpreting the quantum group as a gauge group, one would consider only the invariant elements as "observables." The rest of the algebra would then be an algebra of unobservable fields, whose function in the theory is to describe operations changing the superselection sector ("creating a charge"). Already in the case of Fermi fields one has to relax the requirement of locality for the field algebra (allowing anti-commutation rules). The lack of locality for the action of the quantum group on the "fields" could be seen as a reflection of this general feature of field algebras. Indeed, some constructions of field algebras in this context lead to very non-local objects [BF, Ber].

The theory of superselection sectors in field theory provides some of the most interesting applications of quantum groups. The basic problem is to relate the set of sectors, together with their composition ("fusion") rules, to the set of irreducible representations of a group or quantum group with the rules for decomposing tensor products. In two and more space dimensions this program has been carried out with complete success by Doplicher and Roberts [DR1,DR2], building on earlier work together with Haag [DHR]. They managed to show, in two or more space dimensions, and using only axiomatic assumptions on the observable algebra, that the superselection structure indeed comes from the representation theory of a compact gauge group. They also reconstructed an algebra of fields with an action of the gauge group, whose fixed points are precisely the observables. The fact that they get a (non-quantum) group depends crucially on having more than one space dimension, and hence the possibility of exchanging two spacelike regions in a continuous process during which they always remain spacelike. In one space dimension the superselection structure can be much more complex [MS, SV]. At the same time, there is a rich supply of explicit models with conformal symmetry, for which the superselection structure can be computed (see e.g. [Vec]).

Regarding the connection with the present paper, we wish to point out, however, that quantum groups of the kind we use are not so interesting for the project of reconstructing superselection structures. For example, the irreducible representations of the quantum deformation $\mathrm{S}_{v} \mathrm{U}(2)$ of $\mathrm{SU}(2)$ and the decomposition weights for tensor products are precisely the same as for $\mathrm{SU}(2)$ for real values of the deformation parameter $v$. New features, such as structures with only finitely many sectors, are seen only for complex values of $v$, particularly roots of unity. In that case, however, one loses the involution in the algebra of "functions" on the group, and with it the notion of an action on the observable algebra, which is the object of our investigation.

The decomposition of the algebra of a spin chain with respect to the representations of a group is also reflected in the decomposition theory of invariant states. We discuss one possibility of defining quantum group invariant states even when there is no action: a "hereditarily invariant state" has the property that all its restrictions to finite segments are invariant to the quantum group action given for the segment. Unfortunately, this seems to give no interesting result: on the basis of computations on short chains we conjecture that for $\mathrm{S}_{\nu} \mathrm{U}(2)$ only one state (an infinite product state) has this property. 
In the case of classical groups, there is a general construction [FNW1, FNW2] yielding non-trivial states on a chain which are translationally invariant, and also invariant under the action of the group. These states are automatically ground states of a suitable finite range interaction. The whole construction is naturally covariant, also with respect to quantum groups. It then yields a class of translationally invariant finite range interactions, which are also invariant with respect to an irreducible representation of a quantum group, and have a unique ground state (see [KSZ] for the minimal non-trivial example in this class). Of course, in the case of a classical group the unique ground state is then also invariant under the group. Not so for quantum groups: on the full chain we cannot even say what an invariant state should be, because there is no action of the quantum group. On the half chain, where we can define an action of the quantum group, the uniqueness of the ground state fails, and we get a finite dimensional set of ground states, parametrized by a boundary condition. Among these ground states we now have one state which is translationally invariant, and another state, which is quantum group invariant. Of course, the two are different.

Since our main objective is to point out the difficulties in combining local structure with quantum group symmetry, we have not aimed at maximum generality. The only concrete quantum group we consider is Woronowicz's one-parameter deformation $\mathrm{S}_{v} \mathrm{U}(2)$ of $\mathrm{SU}(2)$. Since this example has served as the paradigm of a quantum group in many papers, we are confident that the difficulties pointed out by us are indeed typical. There are two properties which we prove for $S_{v} U(2)$ (Proposition 4 and Proposition 5) which can be stated for general quantum groups. We would like to pose their generalization to other quantum groups as a challenge to experts in the field. Even in case of $\mathrm{S}_{v} \mathrm{U}(2)$ we had to leave unsettled one statement (Conjecture 8 ), which implies, among other things, that only a specific product state is both quantum group and translationally invariant for the canonical action on the half chain. Much of the literature is phrased in terms of quantum groups in the sense of Drinfel'd rather than Woronowicz. We chose the latter definition because the notion of "action" seemed more natural in this context. The connection between the two approaches is briefly indicated in the Appendix.

The paper is organized as follows. In Sect. 2 we review briefly the notion of quantum group in the sense of Woronowicz [Wo2, Wo4], and of the action of a quantum group on a $C^{*}$-algebra, of fixed points under such an action, and of invariant states with respect to such an action. In Sect. 3 we consider the operation of tensor product for unitary representations and for actions, and describe the basic locality problem for such tensor products. We introduce a more restrictive definition of "actions," which seems more natural for discussing tensor products. Unfortunately, where the standard definition leads to locality problems for extending the chain to the left, the more restrictive definition creates problems right and left. In Sect. 3, we also define the action on a half chain associated with a unitary representation on the one-site Hilbert space, and its construction in terms of an action on the Cuntz algebra $\mathcal{O}_{d}$. In Sect. 4 , we define invariant elements, and show the compatibility of this notion with the local structure, and discuss the hereditarily invariant states. Sect. 5 contains the NO-GO Theorem for actions on the quasi-local algebra, and Sect. 6 discusses similar problems for the implementation of actions by unitaries in the GNS representation of an invariant state. In Sect. 7, we discuss the quantum group covariance of the construction of finitely correlated states. The decay rate in such a state is given by a quantum Wigner $6 j$-symbol. These have been computed in detail [Rue, Bie, LB], so, in principle, we can save ourselves the work of 
diagonalizing a transfer operator. In the explicit example of the q-AKLT model, however, diagonalizing the transfer matrix directly by hand is so straightforward that checking the conventions used in any particular computation of Wigner $6 j$ symbols would not be worth the effort.

In order to do some of the more tedious quantum group computations reliably, we developed a package for Mathematica [Mat], which is available by anonymous ftp from nostromo.physik.Uni-Osnabrueck.de.

\section{Quantum Groups}

As there is not yet a standard notion of quantum group (also called pseudogroup) and of the related invariance and covariance properties, we will briefly review how quantization works for the case of compact groups $\mathscr{G}$. Our discussion will be based completely on the notion of quantum groups introduced by Woronowicz [Wo2, Wo4, Wo1, Wo3]. An alternative would be the Drinfel'd approach [Dri, Jim], which provides a "quantization" of Lie algebras rather than groups. Some of the questions considered in this paper could also be posed using this approach, but we found the Woronowicz approach more suited for this purpose. On the other hand, the Drinfel'd approach is much more effective for doing explicit computations. Therefore, for the reader's convenience, we have included a brief Appendix on the connection of these approaches. A new approach to quantum groups has recently been initiated by Baaj and Skandalis [BS]. In this context actions on $C^{*}$ algebras have been considered by Cuntz [Cu2].

The topology of a compact group $\mathscr{G}$ is encoded in the algebra $\mathscr{C}(\mathscr{G})$ of continuous, complex-valued functions on the group. This is a $*$-algebra under the natural notions of addition, multiplication and complex conjugation. Equipped with the supremum norm, $\mathscr{C}(\mathscr{G})$ becomes a commutative $C^{*}$-algebra with identity. That this algebra carries the complete information about $\mathscr{G}$ as a topological space, is the content of the "Gel'fand Isomorphism Theorem" which reconstructs, starting from any commutative $C^{*}$-algebra, the compact space on which this algebra is the algebra of continuous functions. The next step is to encode the multiplication operation of $\mathscr{G}$. Three maps are naturally connected to the composition law in $\mathscr{G}$, the existence of a neutral element $e \in \mathscr{G}$ and of the inverse $g^{-1}$ of any $g \in \mathscr{G}$, respectively. These three maps become, in turn,

i) the coproduct $\Delta$ which maps $\mathscr{C}(\mathscr{G})$ into $\mathscr{C}(\mathscr{G} \times \mathscr{G}) \cong \mathscr{C}(\mathscr{G}) \otimes \mathscr{C}(\mathscr{G})$, the complex continuous functions in two variables:

$$
(\Delta f)\left(g_{1}, g_{2}\right)=f\left(g_{1} g_{2}\right), \quad f \in \mathscr{C}(\mathscr{G}), \quad g_{1}, g_{2} \in \mathscr{G} .
$$

ii) The antipode $\kappa$ which maps $\mathscr{C}(\mathscr{G})$ into itself, given by:

$$
\kappa(f)(g)=f\left(g^{-1}\right), \quad f \in \mathscr{C}(\mathscr{G}), \quad g \in \mathscr{G} .
$$

iii) The counit $\varepsilon$ which is the character

$$
\varepsilon(f)=f(e) \quad f \in \mathscr{C}(\mathscr{G}) .
$$

The group-axioms are reflected in the properties of the maps $\Delta, \kappa$ and $\varepsilon$ :

i) Associativity of the composition law in $\mathscr{G}$ :

$$
(\Delta \otimes \mathrm{id}) \circ \Delta=(\mathrm{id} \otimes \Delta) \circ \Delta .
$$


ii) $e$ is the neutral element in $\mathscr{G}$ :

$$
(\varepsilon \otimes \mathrm{id}) \circ \Delta=(\mathrm{id} \otimes \varepsilon) \circ \Delta=\mathrm{id} .
$$

iii) $g^{-1}$ is the inverse of $g$ in $\mathscr{G}$ :

$$
\mathbf{m}((\mathrm{id} \otimes \kappa) \circ \Delta)=\mathbf{m}((\kappa \otimes \mathrm{id}) \circ \Delta)=\varepsilon \mathbb{1},
$$

where $\mathbf{m}$ is the multiplication map from $\mathscr{C}(\mathscr{G}) \otimes \mathscr{C}(\mathscr{G}) \rightarrow \mathscr{C}(\mathscr{G})$ taking $f \otimes g$ into $f g$.

We could now consider an abelian algebra that comes with such maps $\Delta, \kappa$ and $\varepsilon$ and reconstruct the compact group $\mathscr{G}$. The key point is, however, that we have nowhere used the commutativity of $\mathscr{C}(\mathscr{G})$, so we can drop this assumption, and arrive at the more general notion of quantum groups. In dropping the commutativity assumption problems arise with the boundedness of $\kappa, \varepsilon$ and $\mathbf{m}$. The multiplication map m on $\mathscr{B}(\mathscr{H}) \otimes \mathscr{B}(\mathscr{H})$, for instance, has norm $\operatorname{dim} \mathscr{H}$ (consider the unitary flip operator $F \varphi \otimes \psi=\psi \otimes \varphi$ on $\mathscr{B}(\mathscr{H}) \otimes \mathscr{B}(\mathscr{H})$, for which $\|\mathbf{m}(F)\|=\operatorname{dim} \mathscr{H})$. The following definition, due to Woronowicz [Wo4], takes care of this difficulty:

1. Definition. $A$ compact quantum group $(\mathscr{C}, \Delta)$ consists of:

i) a separable $C^{*}$-algebra $\mathscr{C}$ with identity $\mathbb{1}$ and

ii) a unital *-homomorphism $\Delta: \mathscr{C} \rightarrow \mathscr{C} \otimes_{\min } \mathscr{C}$,

such that

i) $(\Delta \otimes$ id $) \circ \Delta=($ id $\otimes \Delta) \circ \Delta$, and

ii) both $\Delta(\mathscr{C})(\mathbb{1} \otimes \mathscr{C})$ and $\Delta(\mathscr{C})(\mathscr{C} \otimes \mathbb{1})$ are dense in $\mathscr{C} \otimes_{\min } \mathscr{C}$.

It is shown in [Wo4] that there exists a dense $*$-subalgebra $\mathscr{C}_{0}$ of $\mathscr{C}$ such that $\Delta\left(\mathscr{C}_{0}\right) \subset \mathscr{C}_{0} \odot \mathscr{C}_{0}$, where $\odot$ denotes the algebraic tensor product of $\mathscr{C}_{0}$ with itself, i.e. the finite linear combinations of elements of the form $a \otimes b, a, b \in \mathscr{C}_{0}$. Furthermore $\mathscr{C}_{0}$ is a Hopf $*$-algebra. This means that there are, uniquely determined maps, $\kappa$ and $\varepsilon$, such that:

i) $\kappa$ is a linear, antimultiplicative map from $\mathscr{C}_{0}$ into itself that satisfies $\kappa\left(\left(\kappa\left(a^{*}\right)\right)^{*}\right)=a, a \in \mathscr{C}_{0}$ and

ii) $\varepsilon$ is a $*$-preserving character on $\mathscr{C}_{0}$.

The maps $\Delta, \kappa$ and $\varepsilon$ satisfy Eqs. (2.1), (2.2) and (2.3). The dense $*$-subalgebra $\mathscr{C}_{0}$ consists of all matrix elements of the finite-dimensional unitary representations of $(\mathscr{C}, \Delta)$ (the notion of unitary representation will be introduced shortly).

The standard example of such a structure is the one-parameter deformation $\mathrm{S}_{v} \mathrm{U}(2)$ of $\mathrm{SU}(2)$. Such a deformation is rather drastic in so far that the commutative algebra of complex functions on SU(2) is replaced by an algebra $\mathscr{C}$ with trivial center (see Proposition 4). Still, the whole representation theory of $\mathrm{S}_{v} \mathrm{U}(2)$ turns out to depend smoothly on $v$.

Example. Let $-1 \leqq v \leqq 1$ and $\mathscr{C}$ be the $C^{*}$-algebra with unit, generated by $\alpha$ and $\gamma$, which satisfy the relations:

$$
\begin{aligned}
\alpha \alpha^{*}+v^{2} \gamma^{*} \gamma & =\mathbb{1}, \\
\alpha^{*} \alpha+\gamma \gamma^{*} & =\mathbb{1}, \\
\alpha \gamma^{*}-v \gamma^{*} \alpha & =0 .
\end{aligned}
$$


The relations

$$
\gamma \gamma^{*}=\gamma^{*} \gamma \quad \text { and } \quad \alpha \gamma=v \gamma \alpha
$$

follow automatically [JSW]. The coproduct, antipode and counit are determined by:

$$
\begin{array}{rlrl}
\Delta \alpha=\alpha \otimes \alpha-v \gamma^{*} \otimes \gamma, & & \Delta \gamma=\gamma \otimes \alpha+\alpha^{*} \otimes \gamma, \\
\kappa(\alpha)=\alpha^{*}, & \kappa(\gamma)=-v \gamma, \\
\varepsilon(\alpha)=\mathbb{1}, & \varepsilon(\gamma)=0 .
\end{array}
$$

The relations between $\alpha$ and $\gamma$ are such that

$$
u=\left(\begin{array}{cc}
\alpha & -v \gamma^{*} \\
\gamma & \alpha^{*}
\end{array}\right)
$$

is a unitary in $\mathscr{M}_{2} \otimes \mathscr{C}$. This $u$ is called the fundamental representation of $\mathrm{S}_{v} \mathrm{U}(2)$.

In order to define representations of quantum groups and to construct products of representations two new products are introduced. Let $\mathscr{A}, \mathscr{B}$ and $\mathscr{C}$ be $C^{*}$-algebras with units. We put for $A \in \mathscr{A}, B \in \mathscr{B}$ and $C_{1}, C_{2} \in \mathscr{C}$ :

$$
\begin{aligned}
\left(A \otimes C_{1}\right) \oplus\left(B \otimes C_{2}\right) & =A \otimes B \otimes C_{1} C_{2} \\
& =\left(A \otimes C_{1} \otimes \mathbb{1}_{\mathscr{B}}\right)\left(\mathbb{1}_{\mathscr{A}} \otimes B \otimes C_{2}\right),
\end{aligned}
$$

and, for $A_{1}, A_{2} \in \mathscr{A}$ and $C_{1}, C_{2} \in \mathscr{C}$ :

$$
\left(A_{1} \otimes C_{1}\right) \oplus\left(A_{2} \otimes C_{2}\right)=A_{1} A_{2} \otimes C_{1} \otimes C_{2}
$$

It should be stressed that both $(1)$ and (1) involve an ordinary product in a noncommutative algebra. Therefore the order of the factors is quite important and also the *-operation will behave badly with respect to these products. Although the multiplication map m: $\mathscr{C} \otimes \mathscr{C} \rightarrow \mathscr{C}$ is not bounded on an infinite dimensional nonabelian algebra $\mathscr{C}$, the norm estimate $\|X \oplus Y\| \leqq\|X\|\|Y\|$ holds.

We can now define the analogues of many concepts of classical group theory. In each case it is easy to verify that for abelian $\mathscr{C}$, that is for an ordinary group, the new concept coincides with the ordinary one. When there is a possibility of confusion, we will denote the unit element of an algebra $\mathscr{A}$ by $\mathbb{1}_{\mathscr{A}}$, and the identity map on $\mathscr{A}$ by $\mathrm{id}_{\mathscr{A}}$.

$\triangle$ A unitary representation $v$ of a quantum group $(\mathscr{C}, \Delta)$ on a Hilbert space $\mathscr{H}$ is a unitary element $v \in \mathscr{B}(\mathscr{H}) \otimes \mathscr{C}$ such that $v \oplus v=($ id $\otimes \Delta)(v)$. Suppose that $\mathscr{H}$ is $k$-dimensional and let $\left\{f_{i j} \mid i, j=1,2, \ldots k\right\}$ be matrix units in $\mathscr{M}_{k}(\mathbb{C})$. $v$ can then be written as:

$$
v=\sum_{i j} f_{i j} \otimes v_{\imath j}, \quad v_{\imath j} \in \mathscr{C} .
$$

We can thus consider the $v_{i j}$ as the elements of a $\mathscr{C}$-valued matrix, and identity $\mathscr{M}_{k}(\mathbb{C}) \otimes \mathscr{C}$ with $\mathscr{M}_{k}(\mathscr{C})$, the $\mathscr{C}$-valued $k \times k$-matrices. In terms of the $v_{i j}$ the representation condition is:

$$
\Delta\left(v_{i j}\right)=\sum_{l=1}^{k} v_{i l} \otimes v_{l j} .
$$


$\triangleright$ A linear operator $W: \mathscr{H}_{1} \rightarrow \mathscr{H}_{2}$ intertwines between the unitary representations $v_{1}$ and $v_{2}$ of $(\mathscr{C}, \Delta)$ on $\mathscr{H}_{1}$ and $\mathscr{H}_{2}$ if $\left(W \otimes \mathbb{1}_{\mathscr{C}}\right) v_{1}=v_{2}\left(W \otimes \mathbb{1}_{\mathscr{C}}\right)$.

$\triangleright$ A unitary representation $v$ of $(\mathscr{C}, \Delta)$ is irreducible if the only intertwiners between $v$ and $v$ are the multiples of the identity.

$\triangleright$ A state $h$ on $\mathscr{C}$ is called a Haar measure if:

$$
(h \otimes \mathrm{id}) \circ \Delta=(\mathrm{id} \otimes h) \circ \Delta=h .
$$

In this formula the right-hand side is to be read as the map taking $a \in \mathscr{C}$ to $h(a) \mathbb{1}_{\mathscr{L}}$.

$\triangleright$ A unitary representation $v$ of $(\mathscr{C}, \Delta)$ on $\mathscr{H}$ implements an action $\alpha_{v}$ of $(\mathscr{C}, \Delta)$ on $\mathscr{B}(\mathscr{H})$ by restricting $\operatorname{ad}(v)$ to $\mathscr{B}(\mathscr{H}) \otimes \mathbb{1}_{\mathscr{C}}$ :

$$
\alpha_{v}(A)=\operatorname{ad}(v)\left(A \otimes \mathbb{1}_{\mathscr{C}}\right)=v\left(A \otimes \mathbb{1}_{\mathscr{C}}\right) v^{*}, \quad A \in \mathscr{B}(\mathscr{H}) .
$$

More generally, an action of a quantum group $(\mathscr{C}, \Delta)$ on a $C^{*}$-algebra $\mathscr{A}$ is a $*$-homomorphism $\alpha$ of $\mathscr{A}$ into $\mathscr{A} \otimes_{\min } \mathscr{C}$ mapping the identity of $\mathscr{A}$ into that of $\mathscr{A} \otimes \mathscr{C}$ and such that:

$$
\left(\alpha \otimes \mathrm{id}_{\mathscr{C}}\right) \circ \alpha=\left(\mathrm{id}_{\mathscr{A}} \otimes \Delta\right) \circ \alpha .
$$

$\triangleright$ We will say that a state $\omega$ on $\mathscr{A}$ is invariant under an action $\alpha$ of $(\mathscr{C}, \Delta)$ on $\mathscr{A}$ if for all $A \in \mathscr{A},\left(\omega \otimes \mathrm{id}_{\mathscr{C}}\right)(\alpha(A))=\omega(A) \mathbb{1}_{\mathscr{b}}$.

Let us show the existence of invariant states for a quantum group with a Haar measure $h$ acting on a $C^{*}$-algebra $\mathscr{A}$ by $\alpha$. For any state $\omega$ on $\mathscr{A}$, define the average $\bar{\omega}$ over the group by: $\bar{\omega}(A)=(\omega \otimes h)(\alpha(A)), A \in \mathscr{A}$. A simple computation shows that $\bar{\omega}$ is $\alpha$-invariant:

$$
\begin{aligned}
\left(\bar{\omega} \otimes \operatorname{id}_{\mathscr{C}}\right)(\alpha(A)) & =\left(\omega \otimes h \otimes \mathrm{id}_{\mathscr{C}}\right)\left(\left(\alpha \otimes \mathrm{id}_{\mathscr{C}}\right)(\alpha(A))\right) \\
& =\left(\omega \otimes h \otimes \mathrm{id}_{\mathscr{C}}\right)\left(\left(\mathrm{id}_{\mathscr{A}} \otimes \Delta\right)(\alpha(A))\right) \\
& =\left(\omega \otimes\left(h \otimes \mathrm{id}_{\mathscr{C}}\right) \circ \Delta\right)(\alpha(A)) \\
& =(\omega \otimes h)(\alpha(A)) \\
& =\bar{\omega}(A) .
\end{aligned}
$$

Unitary representations and actions on $C^{*}$-algebras are special cases of "linear representations on a vector space." Yet the definitions look slightly different: we took a unitary representation as an element $v \in \mathscr{B}(\mathscr{H}) \otimes \mathscr{C}$, and an action as a map $\alpha: \mathscr{A} \rightarrow \mathscr{A} \otimes \mathscr{C}$. The classical intuition for all representations is that under an action (or representation) $R$ the vector $x \in X$ becomes a function on the group with values in $X$, i.e. an element of $X \otimes \mathscr{C}$. Thus a representation is a map $R: X \rightarrow X \otimes \mathscr{C}$, and the compatibility with the product becomes encoded in the relation

$$
\left(R \otimes \mathrm{id}_{\mathscr{C}}\right) \circ R=\left(\mathrm{id}_{X} \otimes \Delta\right) \circ R .
$$

Of course, when $X$ is finite dimensional, we can set $\operatorname{Re}_{l}=\sum_{j} e_{j} \otimes R_{j l}$, where $R_{j l} \in \mathscr{C}$ satisfy (2.9). This is the $\mathscr{C}$-valued matrix we used for the definition of unitary representations. The difference between unitary representations on Hilbert spaces and actions on $C^{*}$-algebras is thus mainly in the structure of the underlying space and the sense in which it is preserved by the representation: unitarity is most conveniently formulated in terms of $v \in \mathscr{B}(\mathscr{H}) \otimes \mathscr{C}$, whereas the homomorphism property is more easily expressed in terms of $\alpha: \mathscr{A} \rightarrow \mathscr{A} \otimes \mathscr{C}$. 
For the definition of tensor products it is important to apply a representation not only to $X$ (i.e. to "group independent vectors," but also to vectors $x \in X \otimes \mathscr{C}$ which already depend on a group element. Thus we also need to consider maps

$$
\widehat{R}: X \otimes \mathscr{C} \rightarrow X \otimes \mathscr{C} .
$$

In the classical case, when $\mathscr{C}=\mathscr{C}(G)$, we can define $\widehat{R}$ in terms of $R$. In order to do this, we identify $X \otimes \mathscr{C}$ with the algebra of $X$-valued continuous functions on $G$, and set, for continuous $x: G \rightarrow X$,

$$
(\widehat{R} x)(g)=R_{g}(x(g)) \text {. }
$$

More abstractly, this can be written as

$$
\widehat{R}(x \otimes C)=R(x) \mathrm{id}_{X} \otimes C,
$$

where the product on the left is shorthand for $\left(x \otimes C^{\prime}\right)\left(\operatorname{id}_{X} \otimes C\right)=x \otimes\left(C^{\prime} C\right)$. Equation (2.14) makes sense in the quantum group case as well, and one readily verifies that the representation relation for $\widehat{R}$ becomes

$$
\left(\widehat{R} \otimes \mathrm{id}_{2}\right) \circ\left(\mathrm{id}_{1} \otimes \widehat{R}\right) \circ\left(\mathrm{id}_{\mathscr{A}} \otimes \Delta\right)=\left(\mathrm{id}_{\mathscr{A}} \otimes \Delta\right) \circ \widehat{R} .
$$

This is an equation between maps $X \otimes \mathscr{C} \rightarrow X \otimes \mathscr{C} \otimes \mathscr{C}$, and the subscripts 1 and 2 of the identity maps refer to the first and second tensor factor $\mathscr{C}$. Tensor factors $X$ and $\mathscr{C}$ have to be reshuffled but the order of the $\mathscr{C}$ factors is kept unchanged.

From these considerations it seems that the view of an action as a map $\widehat{R}$ on $X \otimes \mathscr{C}$ satisfying (2.16) is simply equivalent to the general definition in Eq. (2.13). However, this is true only as long as we do not consider additional structures on $X$ : a representation on a Hilbert space $X$ is required to be unitary, and a representation on a $C^{*}$-algebra $X$ is required to be a homomorphism. We have seen that for a unitary representation $R$ we can always pass from $R$ to $\widehat{R}$ by (2.15). However, for actions on a $C^{*}$-algebra this choice of $\widehat{R}$ destroys the homomorphism property. Therefore, the following definition is needed to single out the good cases.

2. Definition. An extended action of a quantum group $(\mathscr{C}, \Delta)$ on a $C^{*}$-algebra $\mathscr{A}$ is an automorphism $\widehat{\alpha}$ of $\mathscr{A} \otimes_{\min } \mathscr{C}$ such that

$$
\left(\widehat{\alpha} \otimes \mathrm{id}_{2}\right) \circ\left(\mathrm{id}_{1} \otimes \widehat{\alpha}\right) \circ\left(\mathrm{id}_{\mathscr{A}} \otimes \Delta\right)=\left(\mathrm{id}_{\mathscr{A}} \otimes \Delta\right) \circ \widehat{\alpha} .
$$

An action $\alpha: \mathscr{A} \rightarrow \mathscr{A} \otimes_{\min } \mathscr{C}$ is called extendible, if it is the restriction of an extended action to $\mathscr{A} \otimes \mathbb{1}_{\mathscr{C}}$.

One easily verifies that, for any unitary representation $v$, $\operatorname{ad}(v)$ is an extended action, hence any implemented action in the sense of the above definitions is automatically extendible. Of course, any action of a classical group is also extendible. It is not immediately obvious, then, that there are non-extendible actions at all. However, we will give an example below, in Proposition 5, showing that Definition 2 has non-trivial content.

\section{Tensor Products of Representations and Actions}

In this paper we are mainly interested in the action of quantum groups on composite quantum systems, i.e. in actions on a tensor product. Let $\alpha$ and $\beta$ be actions of 
a quantum group $(\mathscr{C}, \Delta)$ on $\mathscr{A}$ and $\mathscr{B}$ respectively. The product of $\alpha$ and $\beta$ should then be a homomorphism from $\mathscr{A} \otimes \mathscr{B}$ into $\mathscr{A} \otimes \mathscr{B} \otimes \mathscr{C}$. Let $B \in \mathscr{B}$. For a nontrivial action, $\beta(B)$ will have components in $\mathscr{C}$ and, as we only know how to act with $\alpha$ on elements of the form $A \otimes \mathbb{1}_{\mathscr{C}}$, we cannot apply $\alpha \otimes \mathrm{id}_{\mathscr{B}}$ to $A \otimes \beta(B), A \in \mathscr{A}$ and $B \in \mathscr{B}$. Therefore the general notion of action as defined in (2.12) is ill-adapted to tensor constructs.

It is clear from the discussion at the end of the previous section what is missing: we need to define actions as operators on $\mathscr{A} \otimes \mathscr{C}$. With this modified definition of actions it is clear how to define tensor products of general representations: let $\widehat{R}: X \otimes \mathscr{C} \rightarrow X \otimes \mathscr{C}$ and $\widehat{S}: Y \otimes \mathscr{C} \rightarrow Y \otimes \mathscr{C}$ be "extended representations" in the sense of Eq. (2.16). Then we set

$$
\widehat{R} \oplus \widehat{S}=\left(\widehat{R} \otimes \mathrm{id}_{Y}\right)\left(\mathrm{id}_{X} \otimes \widehat{S}\right),
$$

with the obvious reshuffling of tensor factors. One then verifies that $\widehat{R} \oplus \widehat{S}$ is indeed again an extended representation. Moreover, it is obvious that if $\widehat{R}$ and $\widehat{S}$ are both $*$-homomorphisms, or unitary, then so is their $(1)$-product. Of course, the definition agrees with the usual tensor product in the abelian case, provided $R$ is extended to $\widehat{R}$ by virtue of Eq. (2.14). Note, however, that $\widehat{R} \oplus \widehat{S}$ and $\widehat{S} \oplus \widehat{R}$ differ not only in the order of the factors $X$ and $Y$, which could be undone by a suitable flip isomorphism, but also by the ordering of the factors in $\mathscr{C}$.

Of course, we can use the extension (2.15) to extend an arbitrary representation $R$ to $\widehat{R}$, and thus define $R \oplus S=\left(\widehat{R} \otimes \mathrm{id}_{Y}\right)\left(\mathrm{id}_{X} \otimes S\right)$ for such representations. This coincides, in fact, with the standard definition of tensor products of unitary representations. It is unsuitable for actions on $C^{*}$-algebras, however, since it would practically never lead to a homomorphism, and hence not to an action in the sense of (2.12).

For unitary representations, say a representation $v \in \mathscr{B}(\mathscr{H}) \otimes \mathscr{C}$, and $w \in \mathscr{B}(\mathscr{K})$ $\otimes \mathscr{C}$, the $(1)$-product can be written out in terms of matrix elements as

$$
(v \oplus w)_{i \mu, j v}=v_{i j} w_{\mu v}
$$

where latin and greek indices run over bases of $\mathscr{H}$ and $\mathscr{K}$, respectively. Obviously, this use of the symbol " $(7$ " is also consistent with the definition given in (2.6).

In the sequel, we will always consider actions $\alpha_{v}$ implemented by a unitary representation $v$. Since such actions are always extendible $\left(\widehat{\alpha}_{v}=\operatorname{ad}(v)\right)$, their $(1)$ product is well defined according to (3.1). If $v$ and $w$ are as in (3.2), we find

$$
\operatorname{ad}(v \oplus w)=\operatorname{ad}(v) \oplus \operatorname{ad}(w) .
$$

The following theorem summarizes the "locality" properties of the tensor product of two representations.

3. Theorem. Let $v$ and $w$ be unitary representations of a quantum group $(\mathscr{C}, \Delta)$ on Hilbert spaces $\mathscr{H}$ and $\mathscr{K}$. Then

$$
\alpha_{v \oplus w}\left(\mathscr{B}(\mathscr{H}) \otimes \mathbb{1}_{\mathscr{K}}\right)=\alpha_{v}(\mathscr{B}(\mathscr{H})) \otimes \mathbb{1}_{\mathscr{K}} \subset \mathscr{B}(\mathscr{H}) \otimes \mathbb{1}_{\mathscr{K}} \otimes \mathscr{C} .
$$

(2) If $B \in \mathscr{B}(\mathscr{K})$ is $\alpha_{w}$-invariant, i.e. $\alpha_{w}(B)=B \otimes \mathbb{1}_{\mathscr{b}}$, then

$$
\alpha_{v \oplus w}\left(\mathbb{1}_{\mathscr{H}} \otimes B\right)=\mathbb{1}_{\mathscr{H}} \otimes B \otimes \mathbb{1}_{\mathscr{C}} .
$$


(3) If $\mathscr{H}$ and $\mathscr{K}$ are finite dimensional and if the quantum group is $S_{v} \mathrm{U}(2)$, then, conversely, $\alpha_{v \oplus w}\left(\mathbb{1}_{\mathscr{H}} \otimes B\right) \in \mathbb{1}_{\mathscr{H}} \otimes \mathscr{B}(\mathscr{K}) \otimes \mathscr{C}$ implies that $B \in \mathscr{B}(\mathscr{K})$ is invariant.

Proof. In case (1) we have

$$
\begin{aligned}
\alpha_{v \oplus w}\left(\mathscr{A} \otimes \mathbb{1}_{\mathscr{K}}\right) & =(v \oplus w)\left(\mathscr{A} \otimes \mathbb{1}_{\mathscr{K}} \otimes \mathbb{1}_{\mathscr{C}}\right)\left(v \oplus_{w}\right)^{*} \\
& =\left(v \otimes \mathbb{1}_{\mathscr{K}}\right)\left(\mathbb{1}_{\mathscr{H}} \otimes w\right)\left(\mathscr{A} \otimes \mathbb{1}_{\mathscr{K}} \otimes \mathbb{1}_{\mathscr{C}}\right)\left(\mathbb{1}_{\mathscr{H}} \otimes w\right)^{*}\left(v \otimes \mathbb{1}_{\mathscr{K}}\right)^{*} \\
& =\left(v \mathscr{A} \otimes \mathbb{1}_{\mathscr{C}} v^{*}\right) \otimes \mathbb{1}_{\mathscr{K}} \\
& \subset\left(\mathscr{A} \otimes \mathbb{1}_{\mathscr{K}} \otimes \mathscr{C}\right) .
\end{aligned}
$$

In case (2):

$$
\begin{aligned}
\alpha_{v \oplus w}\left(\mathbb{1}_{\mathscr{H}} \otimes B\right) & =(v \oplus w)\left(\mathbb{1}_{\mathscr{H}} \otimes B \otimes \mathbb{1}_{\mathscr{C}}\right)(v \oplus w)^{*} \\
& =\left(v \otimes \mathbb{1}_{\mathscr{K}}\right)\left(\mathbb{1}_{\mathscr{H}} \otimes\left(w B \otimes \mathbb{1}_{\mathscr{C}} w^{*}\right)\right)\left(v \otimes \mathbb{1}_{\mathscr{K}}\right)^{*} \\
& =\left(v \otimes \mathbb{1}_{\mathscr{K}}\right)\left(\mathbb{1}_{\mathscr{H}} \otimes B \otimes \mathbb{1}_{\mathscr{C}}\right)\left(v \otimes \mathbb{1}_{\mathscr{K}}\right)^{*} \\
& =\mathbb{1}_{\mathscr{H}} \otimes B \otimes \mathbb{1}_{\mathscr{C}} .
\end{aligned}
$$

(3) It is useful to express the action of $\alpha_{v \oplus w}$ in matrix elements with respect to some bases in $\mathbb{C}^{d}$ and $\mathbb{C}^{k}$. Then $v \in \mathscr{M}_{d}(\mathscr{C})$ has matrix elements $v_{i j} \in \mathscr{C}, i, j=1, \ldots, d$, and $w \in \mathscr{M}_{k}(\mathscr{C})$ has matrix elements $w_{\lambda \mu} \in \mathscr{C}, \lambda, \mu=1, \ldots, k$. Then

$$
\begin{aligned}
\left(\alpha_{v \oplus w}(A \otimes B)\right)_{\imath \lambda, l^{\prime} \lambda^{\prime}} & =\left((v \oplus w)\left(A \otimes B \otimes \mathbb{1}_{\mathscr{C}}\right)(v \oplus w)^{*}\right)_{\iota \lambda,^{\prime} \lambda^{\prime}} \\
& =\sum_{j \mu j^{\prime} \mu^{\prime}} v_{l j} w_{\lambda \mu} A_{j j^{\prime}} B_{\mu \mu^{\prime}}\left(w_{\lambda^{\prime} \mu^{\prime}}\right)^{*}\left(v_{i^{\prime} j^{\prime}}\right)^{*} \\
& =\sum_{j j^{\prime}} v_{i j} A_{j j^{\prime}} X_{i \lambda^{\prime}}\left(v_{l^{\prime} j^{\prime}}\right)^{*}, \\
\text { where } \quad X_{\lambda \lambda^{\prime}} & =\sum_{\mu \mu^{\prime}} w_{i \mu} B_{\mu \mu^{\prime}}\left(w_{\lambda^{\prime} \mu^{\prime}}\right)^{*}=\left(w\left(B \otimes \mathbb{1}_{\mathscr{C}}\right) w^{*}\right)_{\lambda \lambda^{\prime}} \in \mathscr{C} .
\end{aligned}
$$

Suppose that, for some $B \in \mathscr{M}_{k}(\mathbb{C})$, and $A_{j j^{\prime}}=\delta_{j j^{\prime}}$, the above matrix element contains a factor $\delta_{i i^{\prime}}$. We can rewrite this as $\sum_{J} v_{i j} X_{\lambda \lambda^{\prime}}\left(v_{l^{\prime} J}\right)^{*}=\delta_{i i^{\prime}} \widetilde{X}_{\lambda \lambda^{\prime}}$, with $\widetilde{X}_{\lambda \lambda^{\prime}} \in \mathscr{C}$. In basis free formulation this reads $v\left(\mathbb{1}_{d} \otimes X_{\lambda, \lambda^{\prime}}\right) v^{*}=\mathbb{1}_{d} \otimes \widetilde{X}_{\lambda \lambda^{\prime}}$, for all $\lambda, \lambda^{\prime}$. This condition can be considered for each pair $\lambda \lambda^{\prime}$ separately, and yields, in the special case of $\mathrm{S}_{v} \mathrm{U}(2)$, that $X_{\lambda \lambda^{\prime}}=\widetilde{B}_{\lambda \lambda^{\prime}} \mathbb{1}_{\mathscr{C}}$ for some $\widetilde{B}_{\lambda \lambda^{\prime}} \in \mathbb{C}$ (see the proposition below). But then, by applying the counit $\varepsilon$ to the definition of $X$, we find that

$$
\widetilde{B}_{\lambda \lambda^{\prime}}=\varepsilon\left(X_{\lambda \lambda^{\prime}}\right)=\sum_{\mu \mu^{\prime}} \varepsilon\left(w_{\lambda \mu}\right) B_{\mu \mu^{\prime}} \varepsilon\left(\left(w_{\lambda^{\prime} \mu^{\prime}}\right)^{*}\right)=B_{\lambda \lambda^{\prime}} .
$$

Hence $X=\left(w\left(B \otimes \mathbb{1}_{\mathscr{C}}\right) w^{*}\right)=B \otimes \mathbb{1}_{\mathscr{C}}$, i.e. $B$ is invariant under $w$.

The special property of $\mathrm{S}_{v} \mathrm{U}(2)$ used in the proof of (3) is isolated in the following proposition. It is clearly violated for ordinary groups, for which (3) fails accordingly. In a sense it expresses the property that $\mathrm{S}_{\nu} \mathrm{U}(2)$ is "completely quantum." In particular, it shows that the center of $S_{v} U(2)$ consists only of multiples of the identity. 
4. Proposition. Let $v$ be a non-trivial d-dimensional unitary representation of $\mathrm{S}_{v} \mathrm{U}(2)$ with $d<\infty$, and let $X, \widetilde{X} \in \mathrm{S}_{v} \mathrm{U}(2)$ such that

$$
v\left(\mathbb{1}_{d} \otimes X\right) v^{*}=\mathbb{1}_{d} \otimes \tilde{X} .
$$

Then $X=\tilde{X}$ is a multiple of the identity in $\mathrm{S}_{v} \mathrm{U}(2)$.

Proof. $v$ contains a non-trivial irreducible subrepresentation, hence we may assume without loss of generality that $v$ is irreducible, say, the irreducible representation of dimension $d=(2 s+1), s>0$. Moreover, by applying the result to hermitian and skew-hermitian parts, we can assume that $X$, and consequently, $\widetilde{X}$, is hermitian. By multiplying the equation from the right by $v$ we get $v\left(\mathbb{1}_{d} \otimes X\right)=\left(\mathbb{1}_{d} \otimes \widetilde{X}\right) v$, or

for all $i, j$.

$$
v_{\imath j} X=\tilde{X} v_{\imath j}
$$

In order to make use of this condition we have to obtain information about the matrix elements of the $(2 s+1)$-dimensional, or "spin-s"-representation of $\mathrm{S}_{v} \mathrm{U}(2)$. We use the standard notation $|s, m\rangle, m=-s, \ldots, s$ for the basis vectors of this representation. We can realize it as that subrepresentation of the $2 s$-fold tensor product of the defining spin- $\frac{1}{2}$ representation $u$ with itself, which contains the product vectors $\Psi_{+}=\left|\frac{1}{2}, \frac{1}{2}\right\rangle^{\otimes 2 s}$, and $\Psi_{-}=\left|\frac{1}{2},-\frac{1}{2}\right\rangle^{\otimes 2 s}$, and these vectors are identified with $|s, \pm s\rangle$, respectively. Hence

$$
\langle s, s|v| s,-s\rangle=\left\langle\Psi_{+}, u^{\otimes 2 s} \Psi_{-}\right\rangle=\left\langle\frac{1}{2}, \frac{1}{2}|u| \frac{1}{2},-\frac{1}{2}\right\rangle^{2 s}=\gamma^{2 s},
$$

and, similarly,

$$
\begin{aligned}
\langle s,-s|v| s, s\rangle & =(-v)^{2 s} \gamma^{* 2 s}, \\
\langle s, s|v| s,-s+1\rangle & =\text { const } \times \alpha^{*} \gamma^{2 s-1} .
\end{aligned}
$$

Now let $X$ and $\tilde{X}$ be as in the proposition, and hermitian. Then $X \gamma^{2 s}=\gamma^{2 s} \tilde{X}$, and $X \gamma^{* 2 s}=\gamma^{* 2 s} \tilde{X}$, which implies $\gamma^{2 s} X=\tilde{X} \gamma^{2 s}$. Hence $X$ commutes with $\left(\gamma \gamma^{*}\right)^{2 s}$. Similarly, we conclude that $X$ commutes with even powers of $\alpha^{*} \gamma^{2 s-1}$.

The irreducible representations of the $C^{*}$-algebra of $\mathrm{S}_{v} \mathrm{U}(2)$ are well-known [JSW, Wo2]. In particular, one obtains a faithful family of representations $\pi_{\zeta}$, parametrized by a phase $\zeta \in \mathbb{C}$, by starting from a cyclic vector $\Omega \in \mathscr{H}_{\zeta}$ with $\pi_{\zeta}(\alpha) \Omega=0$, and setting

$$
\pi_{\zeta}(\gamma) \pi_{\zeta}\left(\alpha^{* n}\right) \Omega=\zeta v^{n} \pi_{\zeta}\left(\alpha^{* n}\right) \Omega
$$

The mutually orthogonal vectors $\pi_{\zeta}\left(\alpha^{* n}\right) \Omega, n \in \mathbb{N}$ span the representation space $\mathscr{H}_{\zeta}$. Since the spectrum of $\pi_{\zeta}\left(\gamma \gamma^{*}\right)$ is simple, and $\pi_{\zeta}(X)$ commutes with this operator, $\pi_{\zeta}(X)$ is determined by its eigenvalues $\xi_{n}$ via

$$
\pi_{\zeta}\left(X \alpha^{* n}\right) \Omega=\xi_{n} \pi_{\zeta}\left(\alpha^{* n}\right) \Omega .
$$

Since $X$ commutes with even powers of $\alpha^{*} \gamma^{2 s-1}$, it commutes with $\alpha^{* 2}$, and hence $\xi_{n+2}=\xi_{n}$. Hence $\pi_{\zeta}(X)$ is a linear combination of the identity and the unitary operator $U$ determined by

$$
U \pi_{\zeta}\left(\alpha^{* n}\right) \Omega=(-1)^{n} \pi_{\zeta}\left(\alpha^{* n}\right) \Omega
$$


The coefficient of $U$ must be zero, because $U$ is not in the $C^{*}$-algebra generated by $\pi_{\zeta}(\alpha)$ and $\pi_{\zeta}(\gamma)$. To see this, consider the images of $U, \pi_{\zeta}(\alpha)$, and $\pi_{\zeta}(\gamma)$ in the Calkin algebra, i.e. the quotient of $\mathscr{B}(\mathscr{H})$ by the algebra of compact operators. There the compact operator $\pi_{\zeta}(\gamma)$ becomes zero, so $\pi_{\zeta}(\alpha)$ becomes unitary, and the algebra generated by these two becomes abelian. On the other hand, $U \pi_{\zeta}(\alpha)=-\pi_{\zeta}(\alpha) U$, hence the image of $U$ cannot be in this abelian algebra.

Hence $\pi_{\zeta}(X)=f(\zeta) \mathbb{1}$, and we have to show that $f$ is constant. Different representations are connected via $\pi_{\zeta} \circ \Phi_{t}=\pi_{\zeta+t}$, where $\Phi_{t}$ are the automorphisms defined by $\Phi_{t}(\alpha)=\alpha$, and $\Phi_{t}(\gamma)=\exp (i t) \gamma$. The $n^{\text {th }}$ Fourier coefficient of $f$ is determined by the element $X_{n}=(2 \pi)^{-1} \int d t \exp (-i n t) \Phi_{t}(X) \in \mathrm{S}_{v} \mathrm{U}(2)$. Recall that $X$ may be approximated in norm by polynomials $X^{\varepsilon}$ in $\alpha, \gamma$, and their adjoints. Using the relations (2.4) we can bring every approximating polynomial into a form in which no monomial contains both $\gamma$ and $\gamma^{*}$. Then the above integral picks out precisely those terms from any polynomial containing $n$ factors $\gamma$ (or $-n$ factors $\gamma^{*}$ ). Let $X_{n}^{\varepsilon}$ denote the sum of these terms. Since $\pi_{\zeta}(\gamma)$ is a compact operator it follows that $\pi_{\zeta}\left(X_{n}^{\varepsilon}\right)$ is compact for $n \neq 0$, and, by norm approximation, so is $\pi_{\zeta}\left(X_{n}\right)$. On the other hand, $\pi_{\zeta}\left(X_{n}\right)$ is a multiple of the identity, and hence must be zero for $n \neq 0$. It follows that all Fourier coefficients of $f$ except the $0^{\text {th }}$ vanish, and so $f$ is constant.

We now come to the discussion of the consequences of Theorem 3 and of the definition of action. Items (1) and (2) of Theorem 3 can both be used to define structures on infinite systems. Let us fix the algebra $\mathscr{A}=\mathscr{M}_{d}(\mathbb{C})$ as the observable algebra at each site of a lattice system, and a unitary representation $v \in \mathscr{M}_{d}(\mathscr{C}) \equiv$ $\mathscr{A} \otimes \mathscr{C}$ of the quantum group $(\mathscr{C}, \Delta)$. The observable algebra associated with a finite subset $\Lambda$ of the lattice under consideration is then $\mathscr{A}^{\Lambda}=\bigotimes_{i \in \Lambda} \mathscr{A}^{(l)}$, where $\mathscr{A}^{(l)}$ is an isomorphic copy of $\mathscr{A}$. By $\mathbb{1}^{\Lambda}$ we denote the identity element in this algebra. If $\Lambda=\Lambda_{1} \cup \Lambda_{2}$ is the disjoint union of two subregions, we have a canonical isomorphism $\mathscr{A}^{\Lambda} \cong \mathscr{A}^{\Lambda_{1}} \otimes \mathscr{A}^{\Lambda_{2}}$. For $\Lambda_{1} \subset \Lambda_{2}$ we have the inclusion $\mathscr{A}^{\Lambda_{1}} \subset \mathscr{A}^{\Lambda_{2}}$, where the inclusion map is $A \mapsto A \otimes \mathbb{1}^{\Lambda_{2} \backslash \Lambda_{1}}$. For an infinite set $\Lambda$ we can therefore consider the union of all algebras $\mathscr{A}^{\Lambda_{f}}$ for finite $\Lambda_{f} \subset \Lambda$. This algebra carries a natural $\mathrm{C}^{*}$-norm, and we will denote by $\mathscr{A}^{\Lambda}$ the $C^{*}$-inductive limit of the $\mathscr{A}^{\Lambda}$, i.e. completion of the union in this norm. As a special case, we obtain the observable algebra, also called the quasi-local algebra of the infinite lattice system [BR], by taking $\Lambda$ as the whole lattice.

In order to define a quantum group action on $\mathscr{A}^{\Lambda}$ we begin with the case of finite $\Lambda$. The unitary representation $v \oplus v \cdots \oplus v$ ( $n$ times $)$ is easily seen to be independent of the bracketing of the $(1)$-products, hence we can define the action $\alpha_{\left(v()^{\cdots} \cdots \Phi^{v)}\right.}$ on $\mathscr{A}^{[1, n]}$. Note that, in contrast to the case of ordinary groups, the ordering of sites in this product is essential, since it fixes the ordering of factors in $\mathscr{C}$. This means that actions of quantum groups can only be defined on one-dimensional lattice systems. Analogously, in quantum field theory, the typical applications of quantum groups are to systems in one space and one time dimension.

In order to define an action on a quasi-local algebra, we have to use the inductive limit process. Thus we would like to define $\alpha(A)=\alpha_{(v \oplus \cdots \oplus)}(A)$, whenever $A \in \mathscr{A}^{[m+1, m+n]}$, i.e. $A$ is in an algebra belonging to $n$ consecutive sites. This preliminary definition has to be checked for consistency with the inclusion maps $A \mapsto A \otimes \mathbb{1}^{\Lambda_{2} \backslash \Lambda_{1}}$, i.e. we have to verify that we obtain the same result if we consider $A$ as an element of a larger algebra $\mathscr{A}^{[m+1-l, m+n+r]}$ with $l, r \geqq 0$. This is precisely the function of Theorem 3(1): it shows that consistency holds for arbitrary $r$ and $l=0$. On the other hand, Theorem 3(3) shows that for a proper quantum group 
consistency fails on the left, i.e. for $l>0$. The best we can do is therefore to define an action $\alpha_{v}^{\mathbb{N}}$ on the half-infinite chain $\mathscr{A}^{\mathbb{N}}$, setting

$$
\alpha_{v}^{\mathbb{N}}(A)=\alpha_{(v \oplus \cdots \oplus v)}(A) \quad \text { for } A \in \mathscr{A}^{[1, n]} \text {. }
$$

There is a very elegant way of constructing this action [KNW], which also underlines the special role of the half chain in this context: the algebra $\mathscr{A}^{\mathbb{N}}$ with $\mathscr{A}=\mathscr{M}_{d}(\mathbb{C})$ can be considered as the gauge invariant part of the Cuntz algebra $\mathcal{O}_{d}$ [Cul]. This is the algebra generated by $d$ Hilbert space operators $S_{i}, i=1, \ldots, d$ satisfying the relations

$$
\begin{gathered}
S_{i}^{*} S_{j}=\delta_{\imath j} \mathbb{1}, \quad \text { for } i, j=1, \ldots, d, \\
\sum_{i=1}^{d} S_{i} S_{i}^{*}=\mathbb{1} .
\end{gathered}
$$

The algebra generated by such operators is independent of the realization, in the sense that for any $C^{*}$-algebra $\mathscr{A}$, and any elements $\widetilde{S}_{i} \in \mathscr{A}$ satisfying the same relations, there is a unique injective $C^{*}$-homomorphism $\Phi: \mathcal{O}_{d} \rightarrow \mathscr{A}$ such that $\Phi\left(S_{i}\right)=\widetilde{S}_{l}$. In particular, there is a one-parameter automorphism group $\gamma_{t}$ on $\mathcal{O}_{d}$ such that $\gamma_{t}\left(S_{j}\right)=e^{i t} S_{j}$. The fixed point algebra of this action is called the gauge invariant part of $\mathcal{O}_{d}$. It is canonically isomorphic to the half chain algebra $\mathscr{A}^{\mathrm{N}}$, because the operators

$$
S_{i_{1}} S_{i_{2}} \cdots S_{i_{n}} S_{i_{n}}^{*} S_{i_{n-1}}^{*} \cdots S_{i_{1}}^{*} \quad \text { for } i_{v}=1, \ldots, d
$$

satisfy precisely the algebraic relations of the matrix units in $\mathscr{A}^{[1, n]}$. Moreover, these matrix units are compatible with tensoring of identity operators on the right, because in the above expression the sum over $i_{n}$ leaves the corresponding expression for $n^{\prime}=n-1$. The idea of [KNW] for obtaining an action of a quantum group on $\mathscr{A}^{\mathbb{N}}$ is to define an action $\alpha^{\mathcal{O}_{d}}$ on $\mathcal{O}_{d}$ instead, which restricts to $\mathscr{A}^{\mathbb{N}}$, because the action commutes with $\gamma_{t}$. Given a unitary representation $v \in \mathscr{M}_{d}(\mathscr{C})$ of the quantum group $(\mathscr{C}, \Delta)$, they define

$$
\alpha^{\mathcal{U}_{d}}\left(S_{i}\right)=\sum_{j=1}^{d} S_{j} \otimes v_{j i} \quad \in \mathcal{O}_{d} \otimes \mathscr{C} .
$$

The existence of a unique injective $C^{*}$-homomorphism $\alpha^{\mathbb{C}_{d}}$ with this property follows at once from the universal property of $\mathcal{O}_{d}$, by verifying that the right-hand side satisfies the relations (3.5). By considering the action on matrix units it becomes clear that this action is the same as the one constructed above. An extension of this construction to the doubly infinite chain is impossible, since the identification of the matrix units, and hence of $\mathscr{A}^{\mathbb{N}}$ as the gauge invariant part of $\mathcal{O}_{d}$ breaks down.

We argued at the beginning of this chapter that for the definition of tensor products it is more natural to consider extended actions $\widehat{\alpha}: \mathscr{A} \otimes \mathscr{C} \rightarrow \mathscr{A} \otimes \mathscr{C}$, rather than simple actions $\alpha: \mathscr{A} \rightarrow \mathscr{A} \otimes \mathscr{C}$. The drawback of this approach to the tensor product of actions is again in the issue of locality: with the simpler notion based on tensoring of unitary representations, we had locality problems at the left end of the chain. With the approach based on extended actions, we get problems right and 
left. In particular, the action on the half chain fails to meet the higher standards for extended actions.

\section{Proposition.}

(1) Let $\alpha_{v}^{\mathbb{N}}$ be the action on the half chain associated with the irreducible representation $v$ of $\mathrm{S}_{v} \mathrm{U}(2)$. Then the relative commutant of $\alpha_{v}^{\mathbb{N}}\left(\mathscr{A}^{\mathbb{N}}\right) \subset \mathscr{A}^{\mathbb{N}} \otimes \mathscr{C}$ consists only of multiples of the identity.

(2) The action of $\alpha_{v}^{\mathbb{N}}$ is not extendible.

Proof. Suppose that $X \in \mathscr{A}^{\mathbb{N}} \otimes \mathscr{C}$ commutes with $\alpha_{v}^{\mathbb{N}}\left(\mathscr{A}^{\mathbb{N}}\right)$. We have to show that $X=x \mathbb{1}$. Let $\omega$ be a state on $\mathscr{A}$, and let $\mathbb{E}_{N}: \mathscr{A}^{\mathbb{N}} \otimes \mathscr{C} \rightarrow \mathscr{A}^{\mathbb{N}} \otimes \mathscr{C}$ be the conditional expectation defined by

$$
\mathbb{E}_{N}\left(A_{N} \otimes A^{\prime} \otimes C\right)=\omega^{\otimes \infty}\left(A^{\prime}\right) A_{N} \otimes \mathbb{1} \otimes C,
$$

where the tensor product refers to the decomposition $\mathscr{A}^{\mathbb{N}} \otimes \mathscr{C}=\mathscr{A}^{[1, N]} \otimes \mathscr{A}^{[N, \infty)} \otimes$ $\mathscr{C}$, and $\omega^{\otimes \infty}$ denotes the infinite product state. Then, since $X \in \mathscr{A}^{\mathbb{N}} \otimes \mathscr{C}$, the sequence $X_{N}=\mathbb{E}_{N}(X)$ converges in norm to $X$. Moreover, $X$, and hence $X_{N}$ commutes with $v^{\Phi^{N}}\left(A_{N} \otimes \mathbb{1} \otimes \mathbb{1}_{\mathscr{C}}\right) v^{\Phi^{N *}}$, for $A_{N} \in \mathscr{A}^{[1, N]}$. Since $v^{\Phi^{N}}$ is unitary, this means that $v^{\Phi^{N *}} X_{N} v^{\Phi^{N *}}$ commutes with all $A_{N}$. Hence this element must be in $\mathbb{1}_{N} \otimes \mathscr{A}^{[N, \infty)} \otimes \mathscr{C}$. By definition of the conditional expectation, it is also in $\mathscr{A}^{[1, N]} \otimes \mathbb{1} \otimes \mathscr{C}$. Hence there is some $C_{N} \in \mathscr{C}$ such that

$$
v^{\oplus N *} X_{N} v^{\oplus N *}=\mathbb{1}_{N} \otimes \mathbb{1} \otimes C_{N} .
$$

Using the relation $X_{N}=\mathbb{E}_{N}\left(X_{N+1}\right)$, we find the formula connecting the different $C_{N} \in \mathscr{C}$ :

$$
C_{N}=\omega \otimes \operatorname{id}\left(v\left(\mathbb{1}_{d} \otimes C_{N+1}\right) v^{*}\right) .
$$

We will show, in the special case of $\mathrm{S}_{v} \mathrm{U}(2)$, that this implies $C_{N}=c \mathbb{1}_{\mathscr{C}}$ for all $N$. Then $X_{N}=c \mathbb{1}$, and $X=\lim _{N} X_{N}=c \mathbb{1}$. The non-extendibility (2) of $\alpha_{v}^{\mathbb{N}}$ follows from statement (1): for if $\hat{\alpha} \in \operatorname{Aut}\left(\mathscr{A}^{\mathbb{N}} \otimes \mathscr{C}\right)$ is an automorphism extending $\alpha_{v}^{\mathbb{N}}$, every element of the form $\widehat{\alpha}(\mathbb{1} \otimes C)$ is in the commutant of $\widehat{\alpha}\left(\mathscr{A} \otimes \mathbb{1}_{\mathscr{C}}\right)=\alpha_{v}^{\mathbb{N}}(\mathscr{A})$. Hence by the determination of the commutant, there must be a linear functional $\eta: \mathscr{C} \rightarrow \mathbb{C}$ such that

$$
\widehat{\alpha}(A \otimes C)=\alpha_{v}^{\mathbb{N}}(A) \eta(C),
$$

which clearly contradicts $\alpha$ being an automorphism. Even if we do not insist on the invertibility of $\widehat{\alpha}$, and allow more general homomorphisms satisfying (2.17), we find from (2.17) that $\eta$ must be a one-dimensional representation of the quantum group, i.e. typically $\eta=\varepsilon$. This choice once again contradicts (2.17).

It remains to prove that equation $(*)$ implies that all $C_{N}$ are multiples of the identity, assuming that $v$ is the spin- $s$ representation of $S_{v} \mathrm{U}(2)$. We are free to choose the state $\omega$ for convenience, and we will take $\omega$ as the pure state with highest 3-component of the spin. Then

$$
\begin{aligned}
\Phi(C) & =\omega \otimes \operatorname{id}\left(v\left(\mathbb{1}_{\mathscr{\alpha}} \otimes C\right) v^{*}\right)=\sum_{m=-s}^{+s} v_{s, m} C v_{s, m}^{*} \\
& =\sum_{m=-s}^{+s} \lambda_{m}\left(\alpha^{s+m}\right)^{*}\left(\gamma^{s-m}\right)^{*} C \gamma^{s-m} \alpha^{s+m}
\end{aligned}
$$


where the $\lambda_{m}$ are strictly positive constants. We will evaluate condition $(*)$, i.e. $C_{N}=\Phi\left(C_{N+1}\right)$ in the faithful family of representations $\pi_{\zeta}$ used in the proof of Proposition 4. Thus, denoting orthonormal basis of the representation space by $|n\rangle$, with $n=0,1, \ldots$, and $\Omega=|0\rangle$, we have

$$
\begin{aligned}
\pi_{\zeta}(\alpha)|n\rangle & =\sqrt{1-v^{2 n}}|n-1\rangle, \\
\pi_{\zeta}\left(\alpha^{*}\right)|n\rangle & =\sqrt{1-v^{2 n+2}}|n+1\rangle, \\
\pi_{\zeta}(\gamma)|n\rangle & =\zeta v^{n}|n\rangle .
\end{aligned}
$$

It follows that the matrix element $\langle n|\Phi(C)| m\rangle$ depends only on the matrix elements $\left\langle n^{\prime}|C| m^{\prime}\right\rangle$ with $n^{\prime} \leqq n, m^{\prime} \leqq m$, and $n-m=n^{\prime}-m^{\prime}$. The iteration of $\Phi$ thus breaks down into a family of finite dimensional iterations of a triangular matrix with positive entries. Each of these operators is contractive, and has a unique fixed point, which is zero for $n \neq m$, and a vector with constant entries for $n=m$. Now for each $N, C_{N}=\Phi^{M}\left(C_{N+M}\right)$, with $\left|\left\langle n\left|C_{N+M}\right| m\right\rangle\right| \leqq\|X\|$, for all $n, m$. Since $M$ can be chosen arbitrarily large, each matrix element of $C_{N}$ must be arbitrarily close to a fixed point. Hence $C_{N}$ is a multiple of the identity.

\section{Invariance of Observables and States}

In the previous section we studied the difficulties in extending the notion of group action to an infinite chain. The basic problem was that the family of actions, defined for each finite segment, are not compatible with the identifications used for the $C^{*}$ inductive limit by which the algebra of the whole chain is defined. In this section we will see that some derived structures, defined on finite segments in terms of the quantum group action, may nevertheless be compatible with the inductive limit.

The most important case in point is the notion of invariant elements under the action: by Theorem 3 the locality property $\alpha\left(\mathscr{A}^{\Lambda}\right) \subset \mathscr{A}^{\Lambda} \otimes \mathscr{C}$ does hold for the quantum group invariant elements. This allows us to make the following definition:

6. Definition. Let $\mathscr{A}=\mathscr{M}_{d}(\mathbb{C})$, and $v$ a d-dimensional unitary representation of a quantum group $(\mathscr{C}, \Delta)$. Let $m \in \mathbb{Z}$, and $n \in \mathbb{N}$, and let $A \in \mathscr{A}^{[m+1, m+n]}$. Then $A$ is called $\operatorname{ad}(v)$-invariant, if $A$ is an intertwiner for $v \oplus \cdots \oplus v$, or equivalently, if $\operatorname{ad}(v \oplus \cdots \oplus v)(A)=A \otimes \mathbb{1}_{\mathscr{b}}$.

The point is that this definition is independent of the local algebra in which we consider $A$, i.e. the ad $(v)$-invariance of $A$ implies the invariance of $\mathbb{1}_{. \mathscr{A}} \otimes A$, and $A \otimes \mathbb{1}_{\mathscr{A}}$, which are considered to be "the same element" in the quasi-local algebra. For ordinary groups, the notion of $\operatorname{ad}(v)$-invariance is equivalent to the invariance of $A$ under the action $\operatorname{ad}\left(v^{\otimes \infty}\right)$ on the whole lattice system. However, as we have seen, this action on the whole chain $\mathscr{A}^{\mathbb{Z}}$ is not well-defined in the quantum group case. Thus the invariance in Definition 6 is not the invariance with respect to a fixed action of the quantum group.

The structure of the algebra of invariant elements on a finite chain is determined essentially by the reduction theory of the tensor product representations $v^{\Phi^{n}}$ into irreducible ones. In the case of $\mathrm{S}_{v} \mathrm{U}(2)$ these decompositions are isomorphic to those for the classical group SU(2). Therefore the inductive limits of the algebras of invariant elements are also isomorphic in the deformed and undeformed case. 
The second notion we are interested in is that of invariant states. We saw we cannot define the quantum group invariance of a translation invariant state as invariance under an action, simply because such actions don't exist. However, just as in the case of invariant observables we may define this property by considering only a finite subchain at a time.

7. Definition. Let $\mathscr{A}=\mathscr{M}_{d}(\mathbb{C})$, and $v$ a d-dimensional unitary representation of a quantum group $(C, \Delta)$. Then a state $\omega$ on $\mathscr{A}^{\mathbb{Z}}$ is called hereditarily invariant, if, for all $n \leqq m \in \mathbb{Z}$, the restriction $\omega \uparrow \mathscr{A}^{[n, m]}$ is invariant with respect to $\alpha_{v} \mathbb{\Phi}^{m-n+1}$.

It is easy to see that if $\omega$ is invariant for $\alpha_{v \oplus w}$, its restriction to the first tensor factor is $\alpha_{v}$-invariant. From examples one can see that its restriction to the second factor is not necessarily $\alpha_{w}$-invariant. On the other hand, hereditarily invariant states do exist: if $\omega=\omega_{1} \otimes \omega_{2}$ with $\omega_{1} \alpha_{v}$-invariant, and $\omega_{2} \alpha_{w}$-invariant, then $\omega$ turns out to be $\alpha_{v \Phi^{w}}$-invariant. Hence the infinite product state formed with an $\alpha_{v}$-invariant state at each site of an infinite chain is hereditarily invariant. Since the reduction theory of tensor products, and hence the decomposition rules for invariant states are the same for $\mathrm{S}_{v} \mathrm{U}(2)$ as for $\mathrm{SU}(2)$ one might expect that, as in the classical case, there may be many hereditarily invariant states. However, once more the $v$ deformation spoils this expectation. In explicit computations (spin-1/2 chain up to length 6 , spin-1 chain up to length 3 , and some tensor products of other irreducible representations) we found that only the product state is hereditarily invariant. We were not able, however, to decide the following statement:

8. Conjecture. Let $v$ be an irreducible unitary representation of $\mathrm{S}_{v} \mathrm{U}(2)$. Then the only hereditarily invariant state of $\mathscr{A}^{\mathbb{Z}}$ is the product state $\omega_{1}^{\otimes \infty}$ formed with the $\alpha_{v}$-invariant state $\omega_{1}$ at each single site.

\section{Quasi-Local Actions}

The arguments of the previous paragraphs show that local actions of quantum groups cannot be obtained using the recipes familiar from classical groups. We will now show that these difficulties are inherent in the quantum group concept, i.e. local actions do not exist on general grounds. Of course, there is always the trivial action of a quantum group, which is obviously local. For a classical group we would exclude such trivialities by assuming the action to be faithful, i.e. that the only group element $g$ with $\alpha_{g}(A)=A$ for all $A$ is the identity. The following proposition shows how to say this for the action of a quantum group. The condition of nuclearity is automatically satisfied for the quasi-local algebra of a spin chain. It implies that the minimal and maximal $C^{*}$-tensor products [Tak] of $\mathscr{A}$ with any other $C^{*}$-algebra coincide. In particular, the minimal tensor product $\mathscr{A} \otimes_{\min } \mathscr{C}$ we have been using in the definition of actions is the same as the maximal one.

9. Proposition. Let $\alpha: \mathscr{A} \rightarrow \mathscr{A} \otimes \mathscr{C}$ be an action of a quantum group $(\mathscr{C}, \triangle)$ on a nuclear $C^{*}$-algebra $\mathscr{A}$. Then there is a smallest $C^{*}$-subalgebra $\mathscr{C}_{0} \subset \mathscr{C}$ such that $\alpha(\mathscr{A}) \subset \mathscr{A} \otimes \mathscr{C}_{0} . \mathscr{C}_{0}$ is closed under the coproduct in the sense that $\Delta\left(\mathscr{C}_{0}\right) \subset$ $\mathscr{C}_{0} \otimes \mathscr{C}_{0}$, and is hence a quantum group in its own right. In the classical case $\mathscr{C}=\mathscr{C}(G)$ it is the algebra of functions on the quotient of $G$ by the subgroup of all $h$ such that $\alpha_{h}(A)=A$ for all $A=\mathscr{A}$. 
For the proof we need a fact about nuclear $C^{*}$-algebras, which we summarize in a lemma. When $\rho \in \mathscr{A}^{*}$ is a linear functional, denote by " $\rho \otimes \mathrm{id}_{\mathscr{C}}$ " the continuous linear extension of $\left(\rho \otimes \operatorname{id}_{\mathscr{C}}\right)(A \otimes C)=\rho(A) C$. For a state $\rho$ this is the conditional expectation onto the second factor.

10. Lemma. Let $\mathscr{A}$ be a nuclear $C^{*}$-algebra, $\mathscr{C}$ another $C^{*}$-algebra, and $\mathscr{D} \subset$ $\mathscr{A} \otimes \mathscr{C}$ a closed subspace. Let $\mathscr{C}_{0} \subset \mathscr{C}$ be the closed subspace generated by all elements of the form $\left(\rho \otimes \operatorname{id}_{\mathscr{C}}\right)(D)$, where $\rho \in \mathscr{A}^{*}$, and $D \in \mathscr{D}$. Then $\mathscr{C}_{0}$ is the smallest closed subspace with the property

$$
\mathscr{D} \subset \overline{\mathscr{A} \otimes \mathscr{C}_{0}}\|\cdot\|
$$

Proof of the Lemma. We first show that $\mathscr{C}_{0}$ has the stated property. Since $\mathscr{D}$ and $\overline{\mathscr{A} \otimes \mathscr{C}_{0}}\|\cdot\|$ are norm closed subspaces, the inclusion given is equivalent to

$$
\left(\mathscr{A} \otimes \mathscr{C}_{0}\right)^{\perp} \subset \mathscr{D}^{\perp},
$$

where $\mathscr{D}^{\perp}$ denotes the space of functionals in $(\mathscr{A} \otimes \mathscr{C})^{*}$ annihilating a subspace $\mathscr{D}$. We now show that, for any finite rank operator $F: \mathscr{A}^{*} \rightarrow \mathscr{A}^{*}$, we have

$$
\left(F \otimes \operatorname{id}_{\mathscr{C}}\right)\left(\mathscr{A} \otimes \mathscr{C}_{0}\right)^{\perp} \subset \mathscr{D}^{\perp} .
$$

Since $F$ is of finite rank, it is of the form

$$
\langle F \omega, A\rangle_{\mathscr{A} \cdot \mathscr{L}^{*}}=\sum_{i=1}^{N}\left\langle\omega, X_{i}\right\rangle_{\mathscr{L}^{*} \cdot \mathscr{A}^{* *}}\left\langle\omega_{i}, A\right\rangle_{\mathscr{A}^{*} \cdot \mathscr{A}}
$$

where the brackets denote the canonical bilinear forms of the pairings indicated, and $X_{i} \in \mathscr{A}^{* *}, \omega_{l} \in \mathscr{A}^{*}$. This can also be expressed conveniently as a map $\widetilde{F}$ on density matrices $D_{\omega}$ (defined by $\langle\omega, A\rangle=\operatorname{tr} D_{\omega} \pi(A)$ ) in the universal representation $\pi$ of $\mathscr{A}$ :

$$
\widetilde{F}\left(D_{\omega}\right)=\sum_{l=1}^{N} \operatorname{tr}\left(D_{\omega} X_{i}\right) D_{\omega_{l}},
$$

where $X_{i}$ is now considered as an element of the weak closure of $\pi(\mathscr{A})$. The operator $F \otimes \mathrm{id}_{\mathscr{C}}$ can be expressed similarly by its action on density matrices in the representation $\pi \otimes \pi_{\mathscr{C}}$, where $\pi_{\mathscr{C}}$ is any faithful representation of $\mathscr{C}$. One gets

$$
\left\langle\left(F \otimes \operatorname{id}_{\mathscr{C}}\right) \Omega, Y\right\rangle:=\sum_{i=1}^{N} \operatorname{tr}\left(D_{\Omega} X_{l} \otimes \pi_{\mathscr{C}}\left(\left(\omega_{i} \otimes \mathrm{id}_{\mathscr{C}}\right)(Y)\right)\right)
$$

Now, if $\Omega \in\left(\mathscr{A} \otimes \mathscr{C}_{0}\right)^{\perp}$, and $Y \in \mathscr{D}$, we have $\operatorname{tr}\left(D_{\Omega} X_{i} \otimes \pi_{\mathscr{C}}\left(\omega_{i} \otimes \operatorname{id}_{\mathscr{C}}(Y)\right)\right)=0$ for $X_{i} \in \pi(\mathscr{A})$, and this extends to $X_{l} \in \mathscr{A}^{* *}$, identified with the weak closure of $\pi(\mathscr{A})$. Hence $\left(F \otimes \operatorname{id}_{\mathscr{C}}\right) \Omega \in \mathscr{D}^{\perp}$, which proves $(* *)$.

By nuclearity, the identity on $\mathscr{A}^{*}$ is the simple weak*-limit of a net $F_{\alpha}$ of completely positive normalized, finite rank operators [CE]. Since the $F_{\alpha}$ are uniformly bounded this implies that the identity on $(\mathscr{A} \otimes \mathscr{C})^{*}$ is the limit of the net $\left(F_{\alpha} \otimes \mathrm{id}_{\mathscr{C}}\right)$. That is, for $\Omega \in(\mathscr{A} \otimes \mathscr{C})^{*}$ we have

$$
w^{*}-\lim _{\alpha}\left(F_{\alpha} \otimes \operatorname{id}_{\mathscr{C}}\right) \Omega=\Omega .
$$


Hence, for $\Omega \in\left(\mathscr{A} \otimes \mathscr{C}_{0}\right)^{\perp}$ the preceding paragraph implies $\Omega \in \mathscr{D}^{\perp}$, proving (*). It remains to be shown that $\mathscr{C}_{0}$ is the smallest subspace with the stated property. Suppose that $\mathscr{C}_{1}$ also satisfies $\mathscr{D} \subset{\overline{\mathscr{A} \otimes \mathscr{C}_{1}}}^{\|\cdot\|}$, and let $D \in \mathscr{D}$. Then we can write $D$ as the norm limit of elements

$$
D_{\alpha}=\sum_{i=1}^{N_{\alpha}} A_{\alpha} \otimes C_{\alpha}, \quad \text { with } \quad C_{\alpha} \in \mathscr{C}_{1} .
$$

Thus, for any state $\rho \in \mathscr{A}^{*}$, we get $\left(\rho \otimes \operatorname{id}_{\mathscr{C}}\right)\left(D_{\alpha}\right)=\sum_{i} \rho\left(A_{\alpha}\right) C_{\alpha} \in \mathscr{C}_{1}$, and, since $\left(\rho \otimes \mathrm{id}_{\mathscr{C}}\right)$ is a contraction: $\left(\rho \otimes \mathrm{id}_{\mathscr{C}}\right)(D) \in \mathscr{C}_{1}$. Consequently, $\mathscr{C}_{0} \subset \mathscr{C}_{1}$.

Proof of the Proposition. It is clear that the lemma remains valid, if we demand $\mathscr{C}_{0}$ to be a $C^{*}$-subalgebra rather than a closed subspace. Hence we can take $\mathscr{C}_{0}$ as the $C^{*}$-subalgebra of $\mathscr{C}$ generated by all elements of the form $\left(\rho \otimes \operatorname{id}_{\mathscr{C}}\right)(\alpha(A)) \in \mathscr{C}$. It remains to be shown that $\Delta\left(\rho \otimes \mathrm{id}_{\mathscr{C}}\right) \alpha(A) \subset \mathscr{C}_{0} \otimes \mathscr{C}_{0}$. By the action property of $\alpha$ we have

$$
\begin{aligned}
\Delta\left(\rho \otimes \operatorname{id}_{\mathscr{C}}\right) \alpha(A) & =\Delta(\rho \otimes \mathrm{id}) \alpha(A)=\left(\rho \otimes \operatorname{id}_{\mathscr{C}} \otimes \operatorname{id}_{\mathscr{C}}\right)\left(\operatorname{id}_{\mathscr{A}} \otimes \Delta\right) \alpha(A) \\
& =\left(\rho \otimes \operatorname{id}_{\mathscr{C}} \otimes \operatorname{id}_{\mathscr{C}}\right)\left(\alpha \otimes \operatorname{id}_{\mathscr{C}}\right) \alpha(A)=\left(\left(\rho \otimes \operatorname{id}_{\mathscr{C}}\right) \alpha \otimes \operatorname{id}_{\mathscr{C}}\right) \alpha(A) \\
& \in\left(\left(\rho \otimes \mathrm{id}_{\mathscr{C}}\right) \alpha \otimes \operatorname{id}_{\mathscr{C}}\right) \mathscr{A} \otimes \mathscr{C}_{0} \subset \mathscr{C}_{0} \otimes \mathscr{C}_{0} .
\end{aligned}
$$

Since the $\left(\rho \otimes \operatorname{id}_{\mathscr{C}}\right) \alpha(A)$ generate $\mathscr{C}_{0}$, and $\Delta$ is a ${ }^{*}$-homomorphism, we find $\Delta\left(\mathscr{C}_{0}\right) \subset$ $\mathscr{C}_{0} \otimes \mathscr{C}_{0}$.

When $\mathscr{C}=\mathscr{C}(G)$ is abelian, any $C^{*}$-subalgebra $\mathscr{C}_{0}$ is uniquely characterized by the equivalence relation $g \approx g^{\prime}$ defined by $f(g)=f\left(g^{\prime}\right)$ for all $f \in \mathscr{C}(G)$. In the present case this becomes $\rho\left(\alpha_{g}(A)\right)=\rho\left(\alpha_{g^{\prime}}(A)\right)$ for all $A$ and all $\rho$, i.e. $\alpha_{g}=\alpha_{g^{\prime}}$. Thus $\mathscr{C}_{0}$ is the algebra of functions on the quotient of $G$ by the subgroup acting trivially on $\mathscr{A}$.

Consider now an action on a $C^{*}$-algebra $\mathscr{A}$, containing two "local" subalgebras $\mathscr{A}_{1}$ and $\mathscr{A}_{2}$, by which we only mean in the present context that they commute elementwise. The action is called strictly local, if $\alpha\left(\mathscr{A}_{i}\right) \subset \mathscr{A}_{l} \otimes \mathscr{C}$, which is the notion considered in the previous section. The action is called local, if $\alpha\left(\mathscr{A}_{l}\right) \subset \mathscr{A}_{l}^{\odot} \otimes \mathscr{C}$, where $\mathscr{A}_{l}^{\odot} \supset \mathscr{A}_{l}, i=1,2$, are two algebras which still commute elementwise. The typical situation we have in mind here is that the $\mathscr{A}_{i}$ are the algebras belonging to two disjoint finite regions in the lattice of a spin system, and the $\mathscr{A}_{l}^{\odot}$ belong to two larger, but still disjoint regions. We can consider a still weaker condition, which does not require $\alpha(A)$ to be localized in any finite region, but allows a weak delocalized tail. We call the action quasi-local if, for any localized $A, \alpha(A) \in \mathscr{A}_{\mathrm{ql}} \otimes \mathscr{C}$, where $\mathscr{A}_{\mathrm{ql}}$ denotes the quasi-local algebra of the spin system, i.e. the $C^{*}$-inductive limit of the local algebras. Then by the norm continuity of $\alpha$, we have $\alpha\left(\mathscr{A}_{\mathrm{ql}}\right) \subset \mathscr{A}_{\mathrm{ql}} \otimes \mathscr{C}$. Thus quasi-locality of an action of a quantum group just means that it can be considered as an action on the quasi-local $C^{*}$-algebra.

11. Theorem. Let $\mathscr{A}_{\mathrm{ql}}$ be the quasi-local observables of a spin system on an infinite (not half-infinite) lattice, and let $\alpha: \mathscr{A}_{\mathrm{ql}} \rightarrow \mathscr{A}_{\mathrm{ql}} \otimes \mathscr{C}$ be the action of $a$ quantum group $(\mathscr{C}, \Delta)$ on $\mathscr{A}_{\mathrm{ql}}$. Assume that $\alpha$ is faithful in the sense that $\alpha\left(\mathscr{A}_{\mathrm{ql}}\right) \subset$ $\mathscr{A}_{\mathrm{ql}} \otimes \mathscr{C}_{0}$ holds for no proper $C^{*}$-subalgebra $\mathscr{C}_{0} \subset \mathscr{C}$, and that $\alpha \tau_{x}=\left(\tau_{x} \otimes \mathrm{id}_{\mathscr{C}}\right) \alpha$, for all $x$, where $\tau_{x}$ denotes the automorphism of $\mathscr{A}_{\mathrm{ql}}$ of translation by the lattice vector $x$. Then the $C^{*}$-algebra $\mathscr{C}$ is abelian. 
Proof. For a continuous linear functional $\rho$ on $\mathscr{A}_{\mathrm{ql}}$, and $A \in \mathscr{A}_{\mathrm{ql}}$, consider $(\rho \otimes$ $\left.\operatorname{id}_{\mathscr{C}}\right) \alpha(A) \in \mathscr{C}$ as in the proof of the above lemma. By assumption, elements of this form generate $\mathscr{C}$. Therefore, we only have to show that $\left(\rho \otimes \operatorname{id}_{\mathscr{C}}\right) \alpha(A)$ and $\left(\rho^{\prime} \otimes\right.$ $\left.\operatorname{id}_{\mathscr{C}}\right) \alpha\left(A^{\prime}\right)$ commute for all $A, A^{\prime} \in \mathscr{A}_{\mathrm{ql}}$, and $\rho, \rho^{\prime} \in \mathscr{A}_{\mathrm{ql}}^{*}$. Now, for every $\varepsilon>0$, we can find expressions $\alpha(A)=\sum_{i=1}^{n} A_{i} \otimes C_{l}+\mathbf{R}(\varepsilon)$, and $\alpha\left(A^{\prime}\right)=\sum_{i=1}^{n^{\prime}} A_{i}^{\prime} \otimes C_{i}^{\prime}+\mathbf{R}(\varepsilon)$, where $A_{l}, A_{i}^{\prime} \in \mathscr{A}_{\mathrm{ql}}, C_{i}, C_{i}^{\prime} \in \mathscr{C}$, and here and in the sequel $\mathbf{R}(\varepsilon)$ stands for any rest which is bounded in norm by $\varepsilon$. In these expressions we may take the $A_{l}$ and $A_{i}^{\prime}$ to be localized in a finite subset $\Lambda$ of the lattice. Now let $x$ be a translation such that $\Lambda \cap(\Lambda+x)=\emptyset$, and $\left\|\left[A, \tau_{x} A^{\prime}\right]\right\| \leqq \varepsilon$. Then

$$
\begin{aligned}
{[(\rho} & \left.\left.\otimes \mathrm{id}_{\mathscr{C}}\right) \alpha(A),\left(\rho^{\prime} \otimes \mathrm{id}_{\mathscr{C}}\right) \alpha\left(A^{\prime}\right)\right] \\
& =\sum_{i j} \rho\left(A_{l}\right) \rho^{\prime}\left(A_{j}^{\prime}\right)\left[C_{i}, C_{J}^{\prime}\right]+\mathbf{R}\left(2 \varepsilon\left(\|A\|+\left\|A^{\prime}\right\|+3 \varepsilon\right)\right) \\
& =\sum_{i j} \widetilde{\rho}\left(A_{i} \otimes \tau_{x}\left(A_{j}^{\prime}\right)\right)\left[C_{i}, C_{j}^{\prime}\right]+\mathbf{R}\left(2 \varepsilon\left(\|A\|+\left\|A^{\prime}\right\|+3 \varepsilon\right)\right) \\
& =\left(\widetilde{\rho} \otimes \operatorname{id}_{\mathscr{C}}\right)\left(\left[\alpha(A),\left(\tau_{x} \otimes \operatorname{id}_{\mathscr{C}}\right) \alpha\left(A^{\prime}\right)\right]\right)+\mathbf{R}\left(4 \varepsilon\left(\|A\|+\left\|A^{\prime}\right\|+3 \varepsilon\right)\right) \\
& \left.=\left(\widetilde{\rho} \otimes \operatorname{id}_{\mathscr{C}}\right) \alpha\left(\left[A, \tau_{x} A^{\prime}\right)\right]\right)+\mathbf{R}\left(4 \varepsilon\left(\|A\|+\left\|A^{\prime}\right\|+3 \varepsilon\right)\right),
\end{aligned}
$$

where $\tilde{\rho}$ is a state on $\mathscr{A}_{\mathrm{ql}}$ which coincides with $\rho$ in $\Lambda$ and with $\rho^{\prime} \circ \tau_{-x}$ in $(\Lambda+x)$. Hence $\left\|\left[\left(\rho \otimes \operatorname{id}_{\mathscr{C}}\right) \alpha(A),\left(\rho^{\prime} \otimes \mathrm{id}_{\mathscr{C}}\right) \alpha\left(A^{\prime}\right)\right]\right\| \leqq \varepsilon+4 \varepsilon\left(\|A\|+\left\|A^{\prime}\right\|+3 \varepsilon\right)$, for any $\varepsilon$.

\section{Quasi-Local Actions in a Representation}

The aim of this section is to show the impossibility of constructing in the GNS space of a translation invariant state, genuine quantum group representations, commuting with the shift and sufficiently local.

Let $\omega$ be a translation invariant state on $\mathscr{A}^{\mathbb{Z}}$ and $(\mathscr{H}, \pi, \Omega)$ the corresponding GNS space, representation and cyclic vector. The translation automorphism $\tau$ is implemented by the unitary shift $S$ on $\mathscr{H}$ :

$$
\pi(\tau(X))=S \pi(X) S^{*} \quad \text { with } S \pi(X) \Omega=\pi(\tau(X)) \Omega, \quad X \in \mathscr{A}^{\mathbb{Z}} .
$$

Let $U$ be a unitary representation of a quantum group $(\mathscr{C}, \Delta)$ on $\mathscr{H}$. For $\varphi, \psi \in \mathscr{H}$, $A, C \in \mathscr{B}(\mathscr{H})$ and $B, D \in \mathscr{C}$ we put

$$
\langle A \otimes B \varphi, C \otimes D \psi\rangle=\langle A \varphi, C \psi\rangle B^{*} D,
$$

and extend this bilinearly to $\mathscr{B}(\mathscr{H}) \otimes_{\min } \mathscr{C}$. This is possible because we have for each representation $\tilde{\pi}$ of $\mathscr{C}$ on a Hilbert space $\mathscr{K}$ and for all choices of $\varphi, \psi$ $\in \mathscr{H}, \zeta, \eta \in \mathscr{K}, A_{i}, C_{l} \in \mathscr{B}(\mathscr{H})$ and $B_{i}, D_{l} \in \mathscr{C}, i=1,2, \ldots n, n=1,2, \ldots$,

$$
\begin{aligned}
\left|\left\langle\zeta, \sum_{k, l}\left\langle A_{k} \varphi, C_{l} \psi\right\rangle \tilde{\pi}\left(B_{k}^{*} D_{l}\right) \eta\right\rangle\right| & \mid\left\langle\left(\sum_{k} A_{k} \otimes \tilde{\pi}\left(B_{k}\right)\right) \varphi \otimes \zeta,\right. \\
& \left.\left(\sum_{l} C_{l} \otimes \tilde{\pi}\left(D_{l}\right)\right) \psi \otimes \eta\right\rangle \mid \\
& \leqq\left\|\sum_{k} A_{k} \otimes B_{k}\right\|\left\|\sum_{l} C_{l} \otimes D_{l}\right\|\|\varphi\|\|\psi\|\|\zeta\|\|\eta\| .
\end{aligned}
$$


By $\|A \varphi\|^{2}$ we denote $\langle A \varphi, A \varphi\rangle, \varphi \in \mathscr{H}$ and $A \in \mathscr{B}(\mathscr{H}) \otimes_{\min } \mathscr{C} . U$ is said to act almost locally if for any $\varphi \in \mathscr{H}, \sigma \in \mathscr{C}^{*}, A$ and $B \in \mathscr{A}^{\mathbb{Z}}$,

$$
\lim _{n \rightarrow \infty} \sigma\left(\left\|\left[\pi\left(\tau^{n}(A)\right), U \pi(B) U^{*}\right] \varphi\right\|^{2}\right)=0
$$

12. Proposition. Let $\omega$ be a translation invariant, clustering state on $\mathscr{A}^{\mathbb{Z}}$, with $G N S$ triplet $(\mathscr{H}, \pi, \Omega)$. Let $U$ be a unitary representation of a quantum group $(\mathscr{C}, \Delta)$ on $\mathscr{H}$ which commutes with the shift $S$ on $\mathscr{H}$, acts almost locally and leaves $\Omega$ invariant. Suppose that there is no proper $C^{*}$-subalgebra $\mathscr{C}_{0}$ of $\mathscr{C}$ such that $U \in \mathscr{B}(\mathscr{H}) \otimes \mathscr{C}_{0}$. Then $\mathscr{C}$ is abelian.

Proof. Denoting by $S$ the unitary on $\mathscr{H}$ that implements the shift, we can express clustering as:

$$
\lim _{n \rightarrow \infty}\left\langle\varphi, S^{n} \psi\right\rangle=\langle\varphi, \Omega\rangle\langle\Omega, \psi\rangle .
$$

Choose now $A, B, C, D \in \pi\left(\mathscr{A}^{\mathbb{Z}}\right)$. Using the asymptotic abelianness of $\mathscr{A}^{\mathbb{Z}}, U S=$ $S U, U \Omega=S \Omega=\Omega$ and the almost locality of the action of $U$, we compute:

$$
\begin{aligned}
\left\langle A S^{n} B \Omega, U C S^{n} D \Omega\right\rangle= & \left\langle A\left(S^{n} B\left(S^{*}\right)^{n}\right) \Omega, U C S^{n} D \Omega\right\rangle \\
= & \left\langle A \Omega,\left(S^{n} B^{*}\left(S^{*}\right)^{n}\right) U C S^{n} D \Omega\right\rangle+\mathbf{o}(1) \\
= & \left\langle A \Omega, U S^{n} U^{*} B^{*} U\left(S^{*}\right)^{n} C S^{n} D \Omega\right\rangle+\mathbf{o}(1) \\
= & \left\langle A \Omega, U C S^{n} U^{*} B^{*} U D \Omega\right\rangle \\
& +\left\langle A \Omega, U S^{n}\left[U^{*} B^{*} U,\left(S^{*}\right)^{n} C S^{n}\right] D \Omega\right\rangle+\mathbf{o}(1) \\
= & \left\langle C^{*} U^{*} A \Omega, S^{n} U^{*} B^{*} U D \Omega\right\rangle+\mathbf{o}(1) \\
= & \left\langle C^{*} U^{*} A \Omega, \Omega\right\rangle\left\langle\Omega, U^{*} B^{*} U D \Omega\right\rangle+\mathbf{o}(1) \\
= & \langle A \Omega, U C \Omega\rangle\langle B \Omega, U D \Omega\rangle+\mathbf{o}(1) .
\end{aligned}
$$

Exchanging the roles of $A$ and $B$ and also of $C$ and $D$, replacing $n$ by $-n$, and using the asymptotic abelianness of $\mathscr{A}^{\mathbb{Z}}$ we conclude:

$$
\begin{aligned}
\langle A \Omega, U C \Omega\rangle\langle B \Omega, U D \Omega\rangle & =\lim _{n \rightarrow \infty}\left\langle A S^{n} B \Omega, U C S^{n} D \Omega\right\rangle=\lim _{n \rightarrow-\infty}\left\langle B S^{n} A \Omega, U D S^{n} C \Omega\right\rangle \\
& =\langle B \Omega, U D \Omega\rangle\langle A \Omega, U C \Omega\rangle .
\end{aligned}
$$

But this implies precisely the statement of the proposition.

\section{7. $C^{*}$-Finitely Correlated States}

The basic construction of states on a half chain and a chain that we will use in this section is a generalization of the so-called Valence Bond Solid states [AKLT]. It was first given in [FNW1] and is based on an earlier proposal for the construction of quantum Markov states in [AF]. Apart from quasi-free CAR-states, it is the only construction that we know of for obtaining non-product pure translation invariant states on a spin chain. 
We will assume throughout this section that the single-site observable algebra $\mathscr{A}$ is that of the complex $d \times d$ matrices $\mathscr{M}_{d}(\mathbb{C})=\mathscr{M}_{d}$. A state $\omega$ of the left half chain $\mathscr{A}^{-}=\mathscr{A}^{\mathbb{Z} \backslash \mathbb{N}}$ is completely determined by giving the expectation values $\omega^{[-n,-1]}(A)$ of observables $A \in \mathscr{A}^{[-n,-1]}, n=1,2, \ldots$. The prescription for $\omega^{[-n,-1]}(A)$ must be compatible with the obvious requirement that $\omega^{[-n-1,-1]}(\mathbb{1} \otimes A)=\omega^{[-n,-1]}(A)$. If we are furthermore able to give a construction such that also $\omega^{[-n-1,-1]}(A \otimes \mathbb{1})=$ $\omega^{[-n,-1]}(A)$, then we have in fact defined a translation invariant state on the entire chain by putting $\omega(A)=\omega^{[-n,-1]}(A), A \in \mathscr{A}^{[m-n, m-1]}, m \in \mathbb{Z}, n=1,2, \ldots$.

Let $\mathscr{B}$ be a $*$-subalgebra of the $k \times k$ matrices $\mathscr{M}_{k}$, containing the identity $\mathbb{1}$ of $\mathscr{M}_{k}$ and let $\mathbb{E}$ be a unity preserving, completely positive map from $\mathscr{B} \otimes \mathscr{A}$ to $\mathscr{B}$. Tensoring $\mathbb{E}$ with suitable identity maps on factors $\mathscr{A}$, we can iterate $\mathbb{E}$ to obtain, for $n \in \mathbb{N}$, unity preserving, completely positive maps $\mathbb{E}^{(n)}: \mathscr{B} \otimes \mathscr{A}^{\otimes n} \rightarrow \mathscr{B}$, where

$$
\mathbb{E}^{(n+1)}=\left(\mathbb{E}^{(n)} \otimes \mathrm{id}\right) \circ \mathbb{E}=\left(\mathbb{E} \otimes \mathrm{id}^{\otimes n}\right) \circ \mathbb{E}^{(n)},
$$

and $\mathbb{E}^{(1)}=\mathbb{E}$. Let $\rho$ be a density matrix on $\mathscr{M}_{k}$ and identify $\rho$ with the state $B \in \mathscr{B} \mapsto \operatorname{tr} \rho B$. Given $\mathbb{E}$ and $\rho$, we define a $C^{*}$-finitely correlated state $\omega$ on $\mathscr{A}^{-}$by:

$$
\omega(A)=\rho\left(\mathbb{E}^{(n)}\left(\mathbb{1}_{k} \otimes A\right)\right), \quad A \in \mathscr{A}^{[-n,-1]} .
$$

Subscripts $d$ and $k$ of $\mathbb{1}$ refer to $\mathscr{A}$ and $\mathscr{B}$ respectively. This definition satisfies the compatibility condition $\omega(A)=\omega(\mathbb{1} \otimes A)$ because $\mathbb{E}$ is unity preserving. The Markovian or transfer matrix like character of $C^{*}$-finitely correlated states can be put in evidence by expressing the expectations of elementary tensors as:

$$
\omega\left(A_{-n} \otimes A_{-n+1} \otimes \cdots \otimes A_{-1}\right)=\rho\left(\mathbb{E}_{A_{-1}} \circ \mathbb{E}_{A_{-2}} \circ \cdots \circ \mathbb{E}_{A_{-n}}\left(\mathbb{1}_{k}\right)\right),
$$

$A_{-i} \in \mathscr{A}, i=1,2, \ldots n$. The $\mathbb{E}_{A}$ in this formula are linear transformations of $\mathscr{B}$ given by $\mathbb{E}_{A}(B)=\mathbb{E}(B \otimes A), B \in \mathscr{B}$. We will mostly assume that the triple $(\mathscr{B}, \mathbb{E}, \rho)$ which generates $\omega$ is minimal in the sense that $\rho$ is a faithful state on $\mathscr{B}$ and that $\mathscr{B}$ is the smallest $*$-subalgebra of $\mathscr{M}_{k}$ containing $\mathbb{1}_{k}$ and invariant under the $\mathbb{E}_{A}, A \in \mathscr{A}$.

If $\rho$ satisfies the additional requirement:

$$
\rho=\rho \circ \mathbb{E}_{\mathbb{1}},
$$

then $\omega$ becomes a translation invariant state on the full chain $\mathscr{A}^{\mathbb{Z}}$ by putting

$$
\omega(A)=\rho\left(\mathbb{E}^{(n)}\left(\mathbb{1}_{k} \otimes A\right)\right), \quad A \in \mathscr{A}^{[m, m+n]}, m \in \mathbb{Z} .
$$

A distinctive role is played by the map $\mathbb{E}_{\mathbb{1}}$ because its spectral properties are directly connected to the ergodic properties of $\omega$. In [FNW1, FNW2], it was proven that a $C^{*}$-finitely correlated state $\omega$ is ergodic iff there exists a minimal generating triple $(\mathscr{B}, \mathbb{E}, \rho)$ for $\omega$ such that the eigenvector $\mathbb{1}_{k}$ of $\mathbb{E}_{\mathbb{1}}$ is non-degenerate. $\omega$ is exponentially clustering iff there is a minimal generating triple with trivial peripheral spectrum, meaning that $\mathbb{1}_{k}$ is the only eigenvector of $\mathbb{E}_{\mathbb{1}}$ with an eigenvalue of modulus 1 . For general $C^{*}$-finitely correlated states, it is not known whether minimal generating triples are unique, up to unitary equivalence. Much more can be said if $\omega$ is purely generated, that is, if there is a generating triple $(\mathscr{B}, \mathbb{E}, \rho)$ for $\rho$ with $\mathbb{E}=\operatorname{ad}\left(V^{*}\right)$ where $V$ is an isometry from $\mathbb{C}^{k}$ to $\mathbb{C}^{k} \otimes \mathbb{C}^{d}$. Minimal generating triples are unique in this case and a $C^{*}$-finitely correlated state $\omega$ is pure iff it is purely generated and exponentially clustering. In this case $\mathscr{B}$ will automatically coincide with the full $\mathscr{M}_{k}$. Furthermore, pure $C^{*}$-finitely correlated states arise as the unique ground states of translation invariant, finite range interactions. 
In order to make this connection more explicit we introduce the iterates $V^{(n)}$ of $V . V^{(n)}$ is an isometry from $\mathbb{C}^{k}$ into $\mathbb{C}^{k} \otimes\left(\mathbb{C}^{d}\right)^{\otimes n}$, recursively defined by:

$$
V^{(n+1)}=\left(V \otimes\left(\mathbb{1}_{d}\right)^{\otimes n}\right) V^{(n)}=\left(V^{(n)}=\left(V^{(n)} \otimes \mathbb{1}_{d}\right) V\right.
$$

and $V^{(1)}=V$. The $\mathbb{E}^{(n)}$ are now expressed as $\mathbb{E}^{(n)}=\operatorname{ad}\left(V^{(n) *}\right)$. The reduced $n$-site density matrices $\rho^{[1, n]}$ of $\omega$ can easily be computed. For $A \in \mathscr{A}^{[1, n]}$ :

$$
\omega(A)=\rho\left(V^{(n) *} \mathbb{1}_{k} \otimes A V^{(n)}\right)=\operatorname{tr} \rho\left(V^{(n) *} \mathbb{1}_{k} \otimes A V^{(n)}\right)=\operatorname{tr}\left(V^{(n)} \rho V^{(n) *} \mathbb{1}_{k} \otimes A\right) .
$$

Therefore

$$
\rho^{[1, n]}=\operatorname{tr}_{\mathbb{C}^{k}} V^{(n)} \rho V^{(n) *} .
$$

Let $\left\{e_{1}, \ldots, e_{k}\right\}$ be an orthonormal basis of $\mathbb{C}^{k}$. It is clear from the computation above that, for $0<n$, the reduced density matrix $\rho^{[1, n]}$ will live on the subspace $\mathscr{G}_{n}$ of $\left(\mathbb{C}^{d}\right)^{\otimes n}$ spanned by the vectors $\left\{\varphi_{i j} \mid i, j=1, \ldots, k\right\}$, where:

$$
V^{(n)} e_{j}=\sum_{l=1}^{k} e_{i} \otimes \varphi_{i J} .
$$

Therefore $\rho^{[1, n]}$ is supported by a subspace $\mathscr{G}_{n}$ of $\left(\mathbb{C}^{d}\right)^{\otimes n}$ of dimension at most $k^{2}$, independently of $n$. It can be shown that for $n$ large enough $\operatorname{dim}\left(\mathscr{G}_{n}\right)$ will eventually reach the value $k^{2}$. Let $r$ be the smallest integer such that $\operatorname{dim}\left(\mathscr{G}_{r}\right)=k^{2}$. If we choose as interaction $h \in\left(\mathscr{M}_{d}\right)^{\otimes(r+1)}$ the projection operator in $\left(\mathbb{C}^{d}\right)^{\otimes(r+1)}$ on the orthogonal complement of $\mathscr{G}_{r+1}$ then $\omega\left(\tau^{j}(h)\right)=0$ for all $j \in \mathbb{Z}$. It is therefore a ground state of $H=\sum_{j \in \mathbb{Z}} \tau^{j}(h)$ in a very strong sense as it minimizes even locally the energy. Moreover, it was shown in [FNW1] that $\omega$ is uniquely determined by the conditions $\omega\left(\tau^{j}(h)\right)=0, j \in \mathbb{Z}$. This means that $\omega$ is "locally" exposed by the translates of $h$. The interaction $h$ associated to the pure $C^{*}$-finitely correlated state $\omega$ is often called a VBS interaction.

We will now consider the construction of $C^{*}$-finitely correlated states and of VBS interactions and ground states which are invariant under the action of a quantum group $(\mathscr{C}, \Delta)$. Suppose that we are given unitary representations $v$ and $w$ of $(\mathscr{C}, \Delta)$ on $\mathbb{C}^{d}$ and $\mathbb{C}^{k}$, implementing extended actions $\operatorname{ad}(v)$ and $\operatorname{ad}(w)$ on $\mathscr{A}=\mathscr{M}_{d}$ and on $\mathscr{B} \subset \mathscr{M}_{k}$. A unity preserving completely positive map $\mathbb{E}: \mathscr{B} \otimes \mathscr{A} \rightarrow \mathscr{B}$ is covariant if:

$$
\operatorname{ad}(w) \circ\left(\mathbb{E} \otimes \operatorname{id}_{\mathscr{C}}\right)=\left(\mathbb{E} \otimes \operatorname{id}_{\mathscr{C}}\right) \circ \operatorname{ad}(w \oplus v) .
$$

On the level of the iterates $\mathbb{E}^{(n)}$ of $\mathbb{E}$ covariance becomes:

$$
\operatorname{ad}(w) \circ\left(\mathbb{E}^{(n)} \otimes \operatorname{id}_{\mathscr{C}}\right)=\left(\mathbb{E}^{(n)} \otimes \operatorname{id}_{\mathscr{C}}\right) \circ \operatorname{ad}(w \oplus v \oplus \cdots \oplus v) .
$$

It is instructive to write out the case $n=2$ :

$$
\begin{aligned}
& \operatorname{ad}(w) \circ\left(\mathbb{E}^{(2)} \otimes \mathrm{id}_{\mathscr{C}}\right)=\operatorname{ad}(w) \circ\left(\mathbb{E} \otimes \mathrm{id}_{\mathscr{C}}\right) \circ\left(\mathbb{E} \otimes \mathrm{id}_{\mathscr{A}} \otimes \mathrm{id}_{\mathscr{C}}\right) \\
& =\left(\mathbb{E} \otimes \operatorname{id}_{\mathscr{C}}\right) \circ \operatorname{ad}(w \oplus v) \circ\left(\mathbb{E} \otimes \mathrm{id}_{\mathscr{d}} \otimes \operatorname{id}_{\mathscr{C}}\right) \\
& =\left(\mathbb{E} \otimes \mathrm{id}_{\mathscr{C}}\right) \circ\left(\operatorname{ad}(w) \otimes \mathrm{id}_{\mathscr{A}}\right) \circ\left(\operatorname{id}_{\mathscr{B}} \otimes \operatorname{ad}(v)\right) \circ\left(\mathbb{E} \otimes \mathrm{id}_{\mathscr{A}} \otimes \mathrm{id}_{\mathscr{C}}\right) \\
& =\left(\mathbb{E} \otimes \mathrm{id}_{\mathscr{C}}\right) \circ\left(\operatorname{ad}(w) \otimes \mathrm{id}_{\mathscr{C}}\right) \circ\left(\mathbb{E} \otimes \mathrm{id}_{\mathscr{A}} \otimes \mathrm{id}_{\mathscr{C}}\right) \circ\left(\mathrm{id}_{\mathscr{B}} \otimes \mathrm{id}_{\mathscr{C}} \otimes \operatorname{ad}(v)\right) \\
& =\left(\mathbb{E} \otimes \mathrm{id}_{\mathscr{C}}\right) \circ\left(\mathbb{E} \otimes \mathrm{id}_{\mathscr{L}} \otimes \mathrm{id}_{\mathscr{C}}\right) \circ\left(\operatorname{ad}(w) \otimes \mathrm{id}_{\mathscr{L}} \otimes \mathrm{id}_{\mathscr{L}}\right) \\
& \circ\left(\operatorname{id}_{\mathscr{B}} \otimes \operatorname{ad}(v) \otimes \operatorname{id}_{\mathscr{L}}\right) \circ\left(\operatorname{id}_{\mathscr{B}} \otimes \operatorname{id}_{\mathscr{L}} \otimes \alpha\right) \\
& =\left(\mathbb{E}^{(2)} \otimes \mathrm{id}_{\mathscr{C}}\right) \circ \operatorname{ad}(w \oplus v \oplus v) \text {. }
\end{aligned}
$$


The following proposition shows how shift and quantum group invariance can hold simultaneously for $C^{*}$-finitely correlated states restricted to a half chain, at the cost of introducing an extra tensor factor, however.

13. Proposition. Let $(\mathscr{B}, \mathbb{E}, \rho)$ be a minimal triple generating the $C^{*}$-finitely correlated state $\omega$ such that $\rho=\rho \circ \mathbb{E}_{\mathbb{1}}$ and suppose that the eigenvalue 1 of $\mathbb{E}_{\mathbb{1}}$ is non-degenerate. Let $v$ and $w$ be unitary representations of a quantum group $(\mathscr{C}, \Delta)$ defining actions $\alpha_{v}$ and $\alpha_{w}$ on $\mathscr{A}$ and $\mathscr{B}$ respectively and suppose that $\mathbb{E}$ is covariant.

(1) $\widetilde{\omega}(X)=\rho\left(\mathbb{E}^{(n)}(X)\right), X \in \mathscr{B} \otimes \mathscr{A}^{[0, n-1]}$ defines a state of $\mathscr{B} \otimes \mathscr{A}^{\mathbb{N}}$ and $\widetilde{\omega}$ coincides with $\omega$ on $\mathbb{1}_{\mathscr{B}} \otimes \mathscr{A}^{\mathbb{N}}$.

(2) $\widetilde{\omega}$ is invariant under the action $\left(\operatorname{ad}(w) \otimes \mathrm{id}_{\mathscr{d}} \mathbb{N}\right) \circ \alpha_{v}^{\mathbb{N}}$ on $\mathscr{B} \otimes \mathscr{A}^{\mathbb{N}}$.

Proof. Let $X \in \mathscr{B} \otimes \mathscr{A}^{[0, n-1]}$. We then compute:

$$
\rho\left(\mathbb{E}^{(n+1)}\left(X \otimes \mathbb{1}_{d}\right)\right)=\rho\left(\mathbb{E}\left(\mathbb{E}^{(n)}(X) \otimes \mathbb{1}_{d}\right)\right)=\rho\left(\mathbb{E}^{(n)}(X)\right) .
$$

This is precisely the compatibility condition we need for $\widetilde{\omega}$. Positivity and normalization of $\widetilde{\omega}$ are immediate consequences of the positivity and normalization of $\rho$ and the $\mathbb{E}^{(n)}$. By construction $\widetilde{\omega}$ extends the restriction of $\omega$ to $\mathscr{A}^{\mathbb{N}}$.

We first show that for all $B \in \mathscr{B}$,

$$
\left(\rho \otimes \operatorname{id}_{\mathscr{C}}\right)\left(\alpha_{w}(B)\right)=\rho(B) \mathbb{1}_{\mathscr{C}} .
$$

Consider on $\mathscr{B}$ the functional

$$
B \mapsto \rho \otimes \sigma\left(\alpha_{w}(B)\right)
$$

where $\sigma$ is an arbitrary continuous functional on $\mathscr{C}$. Using the covariance of $\mathbb{E}$ and the invariance of $\rho$ under $\mathbb{E}_{\mathbb{1}}$ we compute:

$$
\begin{aligned}
\rho \otimes \sigma\left(\alpha_{w}\left(\mathbb{E}\left(B \otimes \mathbb{1}_{d}\right)\right)\right) & =\rho \otimes \sigma\left(\operatorname{ad}(w)\left(\left(\mathbb{E} \otimes \mathrm{id}_{\mathscr{C}}\right)\left(B \otimes \mathbb{1}_{d} \otimes \mathbb{1}_{\mathscr{C}}\right)\right)\right) \\
& =\rho \otimes \sigma\left(\left(\mathbb{E} \otimes \operatorname{id}_{\mathscr{C}}\right)\left(\operatorname{ad}(w \oplus v)\left(B \otimes \mathbb{1}_{d} \otimes \mathbb{1}_{\mathscr{C}}\right)\right)\right) \\
& =\rho \otimes \sigma\left(\left(\mathbb{E} \otimes \mathrm{id}_{\mathscr{C}}\right)\left(\alpha_{w}(B) \otimes \mathbb{1}_{d}\right)\right) \\
& =\rho \otimes \sigma\left(\alpha_{w}(B)\right) .
\end{aligned}
$$

By assumption, the eigenvalue 1 of $\mathbb{E}_{\mathbb{1}}$, and therefore also of its dual, is nondegenerate. This implies that for all $\sigma \in \mathscr{C}^{*}$,

$$
\rho \otimes \sigma\left(\alpha_{w}(B)\right)=\rho(B) \sigma\left(\mathbb{1}_{\mathscr{C}}\right)
$$

Therefore

$$
\left(\rho \otimes \operatorname{id}_{\mathscr{C}}\right)\left(\alpha_{w}(B)\right)=\rho(B) \mathbb{1}_{\mathscr{C}}
$$

for $B \in \mathscr{B}$. The invariance of $\widetilde{\omega}$ under $\left(\operatorname{ad}(w) \otimes \mathbb{1}_{\mathscr{d}} \mathbb{N}\right) \circ \alpha_{v}^{\mathbb{N}}$ can now be checked. 
Let $X \in \mathscr{B} \otimes \mathscr{A}^{[0, n-1]}, n=1,2, \ldots$,

$$
\begin{aligned}
\widetilde{\omega} \otimes \operatorname{id}_{\mathscr{C}}\left(\left(\operatorname{ad}(w) \otimes \operatorname{id}_{\mathscr{d}} \mathbb{N}\right)\left(\alpha_{v}^{\mathbb{N}}(X)\right)\right)= & \widetilde{\omega} \otimes \operatorname{id}_{\mathscr{C}}\left(\operatorname{ad}(w \oplus v \oplus \cdots \oplus v)\left(X \otimes \mathbb{1}_{\mathscr{C}}\right)\right) \\
= & \rho \otimes \operatorname{id}_{\mathscr{C}}\left(\left(\mathbb{E}^{(n)} \otimes \operatorname{id}_{\mathscr{C}}\right)\right. \\
& \left.\left(\operatorname{ad}(w \oplus v \oplus \cdots \oplus v)\left(X \otimes \mathbb{1}_{\mathscr{C}}\right)\right)\right) \\
= & \rho \otimes \operatorname{id}_{\mathscr{C}}\left(\operatorname{ad}(w)\left(\mathbb{E}^{(n)}(X) \otimes \mathbb{1}_{\mathscr{C}}\right)\right) \\
= & \rho \otimes \operatorname{id}_{\mathscr{C}}\left(\alpha_{w}\left(\mathbb{E}^{(n)}(X)\right)\right) \\
& =\rho\left(\mathbb{E}^{(n)}(X)\right) \mathbb{1}_{\mathscr{C}} \\
& =\widetilde{\omega}(X) \mathbb{1}_{\mathscr{C}} .
\end{aligned}
$$

The density matrix $\rho$ used in the construction of the $C^{*}$-finitely correlated state $\omega$ in Proposition 13 satisfies $\rho=\rho \circ \mathbb{E}_{\mathbb{1}}$, which is needed in order to insure the translation invariance of the state. It is straightforward to check that there is another choice for the density matrix, namely a density matrix $\rho^{\prime}$ that is invariant under $\operatorname{ad}(w)$, which leads to an $\alpha_{v}^{\mathbb{N}}$ invariant state on $\mathscr{A}^{\mathbb{N}}$. For quantum groups (that are not groups) one should not expect these two requirements, invariance under $\operatorname{ad}(w)$ and $\rho=\rho \circ \mathbb{E}_{\mathbb{1}}$, to be compatible. In the case of the spin $S$ representation of $\mathrm{S}_{v} \mathrm{U}(2), \rho$ and $\rho^{\prime}$ are both unique and coincide only for $v=1$ :

$$
\rho=\frac{1}{[2 S+1]_{v}} v^{-2 J_{z}}, \quad \rho^{\prime}=\frac{1}{2 S+1} \mathbb{1} .
$$

We refer to the Appendix for the notations and the calculation. The conclusion of Theorem 11, that $\alpha_{v}$ and translations are incompatible properties, is then not so surprising.

One should note that, though $\left(\operatorname{ad}(w) \otimes \mathrm{id}_{\mathscr{A}} \mathbb{N}\right) \circ \alpha_{v}^{\mathbb{N}}$ is not a proper action on $\mathscr{A}^{\mathbb{N}}$, still, by Theorem 3(2), if $A \in \mathscr{A}^{\mathbb{N}}$ is invariant under $\alpha_{v}^{\mathbb{N}}, A$ is also invariant under $\left(\operatorname{ad}(w) \otimes \mathrm{id}_{\mathscr{d} / \mathbb{N}} \circ \alpha_{v}^{\mathbb{N}}\right.$. We now consider ground states of spin chains corresponding to $\operatorname{ad}(v)$-invariant VBS interactions. Suppose that we have two unitary representations $v$ and $w$ of $(\mathscr{C}, \Delta)$ on $\mathbb{C}^{d}$ and $\mathbb{C}^{k}$ respectively and an isometry $V: \mathbb{C}^{k} \rightarrow \mathbb{C}^{k} \otimes \mathbb{C}^{d}$ intertwining $w$ and $v \oplus w$, i.e.:

$$
\left(V \otimes \mathbb{1}_{\mathscr{C}}\right) w=(w \oplus v)\left(V \otimes \mathbb{1}_{\mathscr{C}}\right) .
$$

The intertwining property on the level of the $V^{(n)}$ becomes:

$$
\left(V^{(n)} \otimes \mathbb{1}_{\mathscr{C}}\right) w=\left(w \oplus v^{\mathbb{\oplus} n}\right)\left(V^{(n)} \otimes \mathbb{1}_{\mathscr{C}}\right) .
$$

Let $\rho$ be a density matrix on $\mathscr{M}_{k}$ such that

$$
\rho(B)=\rho\left(V^{*}\left(B \otimes \mathbb{1}_{d}\right) V\right), \quad B \in \mathscr{M}_{k} .
$$

Generically, $\rho$ is uniquely determined by this condition and, putting $\mathbb{E}=\operatorname{ad}\left(V^{*}\right), \mathbb{E}_{\mathbb{1}}$ has trivial peripheral spectrum. The $C^{*}$-finitely correlated state generated by $\left(\mathscr{M}_{k}, \mathbb{E}, \rho\right)$ is then a pure, translation-invariant state on the chain $\mathscr{A}^{\mathbb{Z}}$. Let, for $n=1,2, \ldots, \mathscr{G}_{n}$ be the subspaces of $\left(\mathbb{C}^{d}\right)^{\otimes n}$ introduced at the beginning of this section. Recall that $\mathscr{G}_{n}$ is the supporting subspace of the reduced $n$-site density matrix of $\omega$. 
14. Proposition. Let $V: \mathbb{C}^{k} \rightarrow \mathbb{C}^{k} \otimes \mathbb{C}^{d}$ be an isometry, intertwining the unitary representations $v$ and $w$ of the quantum group $(\mathscr{C}, \Delta)$ on $\mathbb{C}^{d}$ and $\mathbb{C}^{k}$ respectively:

$$
\left(V \otimes \mathbb{1}_{\mathscr{C}}\right) w=(w \oplus v)\left(V \otimes \mathbb{1}_{\mathscr{C}}\right) .
$$

The orthogonal projection in $\left(\mathscr{M}_{d}\right)^{\otimes n}$ on the subspace $\mathscr{G}_{n}$ of $\left(\mathbb{C}^{d}\right)^{\otimes n}$ commutes with $v^{\oplus n}, n=1,2, \ldots$.

Proof. Let $\left\{e_{1}, e_{2}, \ldots, e_{k}\right\}$ be an orthonormal basis for $\mathbb{C}^{k}$. The subspace $\mathscr{G}_{n}$ of $\left(\mathbb{C}^{d}\right)^{\otimes n}$ is generated by the vectors $\left\{\varphi_{i j} \mid i, j=1,2, \ldots k\right\}$ with

$$
V^{(n)} e_{j}=\sum_{l=1}^{k} e_{l} \otimes \varphi_{l j}
$$

We can, without loss of generality, assume that $\mathscr{C}$ is a (norm-closed) $*$-subalgebra of the bounded linear operators on some Hilbert space $\mathscr{H}$. Let $\chi \in \mathscr{H}$. We have to show that, for $i, j=1,2 \ldots k, v^{\oplus n} \varphi_{l j} \otimes \chi$ belongs to $\mathscr{G}_{n} \otimes \mathscr{H}$ or, equivalently, that for $j=1,2, \ldots k$,

$$
\left(\mathbb{1}_{k} \otimes v^{(n)}\right)\left(V^{(n)} \otimes \mathbb{1}_{\mathscr{C}}\right) e_{j} \otimes \chi
$$

is an element of $\mathscr{G}_{n} \otimes \mathbb{C}^{k} \otimes \mathscr{H}$,

$$
\begin{aligned}
\left(\mathbb{1}_{k} \otimes v^{\oplus n}\right)\left(V^{(n)} \otimes \mathbb{1}_{\mathscr{C}}\right) e_{j} \otimes \chi & =\left(w^{*} \otimes\left(\mathbb{1}_{d}\right)^{\otimes n}\right)\left(w \oplus v^{\oplus n}\right)\left(V^{(n)} \otimes \mathbb{1}_{\mathscr{C}}\right) e_{j} \otimes \chi \\
& =\left(w^{*} \otimes\left(\mathbb{1}_{d}\right)^{\otimes n}\right)\left(V^{(n)} \otimes \mathbb{1}_{\mathscr{C}}\right) w e_{j} \otimes \chi .
\end{aligned}
$$

This proves the statement as $w^{*}$ acts only in a non-trivial way on $\mathbb{C}^{k} \otimes \mathscr{H}$.

In particular Proposition 14 shows that the VBS interaction corresponding to a pure $C^{*}$-finitely correlated state, generated by an isometric intertwiner of unitary quantum group representations, is $(\mathscr{C}, \Delta)$-invariant. We conclude this section with a discussion of what can be considered to be the simplest possible example of this structure.

Example. Consider the irreducible representations of $\mathrm{S}_{v} \mathrm{U}(2)$ on $\mathbb{C}^{2}$ and $\mathbb{C}^{3}$. This leads to the $q$-deformed AKLT-model as considered in [BY, KSZ] (in the present paper, however, the parameter is called $v$ instead of $q$ ). Instead of using the Woronowicz description, as in the example of Sect. 2, we will turn to the Drinfel'd approach that is much more effective for computations. The connection between both approaches is sketched in the Appendix.

Denote by $[a]_{v}$ the $v$-numbers: $[a]_{v}=\left(v^{a}-v^{-a}\right) /\left(v-v^{-1}\right)$. The commutation relations between the "Lie-algebra generators" of $\mathrm{S}_{v} \mathrm{U}(2)$ are:

$$
\left[J_{z}, J_{ \pm}\right]= \pm J_{ \pm} \quad \text { and } \quad\left[J_{+}, J_{-}\right]=\left[2 J_{z}\right]_{v} .
$$

Product representations are constructed according to the rule

$$
\begin{aligned}
L_{z} & =J_{z} \otimes \mathbb{1}+\mathbb{1} \otimes K_{z}, \\
L_{ \pm} & =v^{J_{z}} \otimes K_{ \pm}+J_{ \pm} \otimes v^{-K_{z}} .
\end{aligned}
$$

There is a quadratic Casimir operator $C$ given by

$$
C=\left[J_{z}+\frac{1}{2}\right]_{v}^{2}-\left[\frac{1}{2}\right]_{v}^{2}+J_{-} J_{+} .
$$


The irreducible representations of $\mathrm{S}_{v} \mathrm{U}(2)$ are completely similar to those of $\mathrm{SU}(2)$. There is, for each $j \in \frac{1}{2} \mathbb{N}$, a unique $(2 j+1)$-dimensional representation labelled by the eigenvalue $[j]_{v}[j+1]_{v}$ of $C$. The explicit forms of the spin $\frac{1}{2}$ and spin 1 representations are:

$$
J_{z}=\left(\begin{array}{cc}
\frac{1}{2} & 0 \\
0 & -\frac{1}{2}
\end{array}\right) \quad J_{+}=\left(\begin{array}{cc}
0 & 1 \\
0 & 0
\end{array}\right) \quad J_{-}=\left(\begin{array}{ll}
0 & 0 \\
1 & 0
\end{array}\right)
$$

and

$J_{z}=\left(\begin{array}{ccc}1 & 0 & 0 \\ 0 & 0 & 0 \\ 0 & 0 & -1\end{array}\right) \quad J_{+}=\left(\begin{array}{ccc}0 & \sqrt{[2]_{v}} & 0 \\ 0 & 0 & \sqrt{[2]_{v}} \\ 0 & 0 & 0\end{array}\right) \quad J_{-}=\left(\begin{array}{ccc}0 & 0 & 0 \\ \sqrt{[2]_{v}} & 0 & 0 \\ 0 & \sqrt{[2]_{v}} & 0\end{array}\right)$.

Denoting by $\left\{\left|\frac{1}{2}\right\rangle,\left|-\frac{1}{2}\right\rangle\right\}$ and $\{|1\rangle,|0\rangle,|-1\rangle\}$ the canonical bases of $\mathbb{C}^{2}$ and $\mathbb{C}^{3}$, the unique intertwiner $V$ between the spin $\frac{1}{2}$ representation and the product of the spin $\frac{1}{2}$ and the spin 1 representation is easily computed:

$$
\begin{aligned}
V\left|\frac{1}{2}\right\rangle & =\frac{1}{\sqrt{[3]_{v}}}\left(v^{-1}\left|\frac{1}{2}, 0\right\rangle-v^{\frac{1}{2}} \sqrt{[2]_{v}}\left|-\frac{1}{2}, 1\right\rangle\right), \\
V\left|-\frac{1}{2}\right\rangle & =\frac{1}{\sqrt{[3]_{v}}}\left(v^{-\frac{1}{2}} \sqrt{[2]_{v}}\left|\frac{1}{2},-1\right\rangle-v\left|-\frac{1}{2}, 0\right\rangle\right) .
\end{aligned}
$$

The $2 \times 2$ density matrix $\rho$, singled out by the invariance condition (7.4), is

$$
\rho=\frac{1}{[2]_{v}}\left(\begin{array}{cc}
v^{-1} & 0 \\
0 & v
\end{array}\right)
$$

and the spectrum of $\mathbb{E}_{\mathbb{1}}$ consist of 1 and $-v^{2} /[3]_{v}$, with degeneracy 3 . The eigenvectors are

$$
\mathbb{1}, \quad J_{z} v^{2 J_{z}}, \quad J_{+} \text {, and } J_{-}, .
$$

Finally, the $C^{*}$-finitely correlated state constructed in this way, is the unique, shiftinvariant ground state of the $v$-invariant, nearest-neighbour, VBS-Hamiltonian on the spin 1 chain, determined by the interaction $h=C^{2}-[2]_{v} C$. Here, the operator $C$ is the Casimir operator in the tensor product of the spin 1 representation with itself. It is, up to a normalization factor, the orthogonal projection onto the spin 2 subrepresentation. For $v=1$ (and up to a multiplicative and additive constant) $h$ reduces to the well-known spin 1 AKLT-interaction $3 \overline{J_{1}} \cdot \overline{J_{2}}+\left(\overline{J_{1}} \cdot \overline{J_{1}}\right)^{2}$, where $\bar{J}$ denotes the three Cartesian components of the spin 1 generators of $\mathrm{SU}(2)$ [AKLT].

\section{A. Drinfel'd Approach to $S_{v} U(2)$}

The purpose of this Appendix is to set up the dual approach to $\mathrm{S}_{v} \mathrm{U}(2)$, which is much more efficient in computations than the Woronowicz version. Nobody in his right mind would do computations concerning representations of $\mathrm{SU}(2)$ using 
the explicit form of the representing unitaries as polynomials in matrix elements of SU(2). Yet this is what the Woronowicz approach requires. Here we provide the associated Lie algebraic version of $\mathrm{S}_{v} \mathrm{U}(2)$, i.e. the corresponding object in the Drinfel'd approach. We present this as a purely computational tool, and leave it to the reader to construct the analogues of the results in the paper in this language.

Throughout, we consider $\mathscr{C}_{0}$, the algebra of polynomials in the generators $\alpha, \gamma$, and their adjoint and not the $C^{*}$-algebra of $\mathrm{S}_{v} \mathrm{U}(2)$. Likewise, tensor products are algebraic tensor products, and the dual $\mathscr{C}_{0}^{*}$ is the algebraic dual. We make $\mathscr{C}_{0}^{*}$ into a Hopf algebra with the operations

$$
\begin{aligned}
\xi \cdot \eta(a) & =(\xi \otimes \eta) \circ \Delta(a), \\
\Delta(\xi)(a \otimes b) & =\xi \circ \mathbf{m}(a \otimes b)=\xi(a b), \\
\mathbb{1}(a) & =\varepsilon(a) .
\end{aligned}
$$

In the classical case there are two important kinds of linear functionals on $\mathscr{C}(G)$ : evaluations at group elements, and directional derivatives at the identity. The latter make up the Lie algebra, and, since $\mathscr{C}_{0}^{*}$ is an algebra, this space is to be considered as the quantization of the universal enveloping algebra, of $\mathrm{S}_{v} \mathrm{U}(2)$, or the quantum group $\mathrm{S}_{v} \mathrm{U}(2)$ in the sense of Drinfel'd. tions

We consider three special functionals $J_{z}, J_{+}, J_{-} \in \mathscr{C}_{0}^{*}$, which satisfy the rela-

$$
\begin{aligned}
& \Delta\left(J_{z}\right)=J_{z} \otimes \mathbb{1}+\mathbb{1} \otimes J_{z}, \\
& \Delta\left(J_{ \pm}\right)=J_{ \pm} \otimes v^{-J_{z}}+v^{J_{z}} \otimes J_{ \pm} .
\end{aligned}
$$

Here the exponential is to be computed using the product in $\mathscr{C}_{0}^{*}$, with the constant term given by the counit. Using (A.2) we can compute these functionals on any polynomials, once they are known on the generators. The following table gives the necessary initial values:

$\begin{array}{cccccc}J(A) & A=\mathbb{1} & A=\alpha & A=\alpha^{*} & A=\gamma & A=\gamma^{*} \\ J=J_{z} & 0 & \frac{1}{2} & -\frac{1}{2} & 0 & 0 \\ J=J_{+} & 0 & 0 & 0 & 0 & -\frac{1}{v} \\ J=J_{-} & 0 & 0 & 0 & 1 & 0\end{array}$

For $J_{z}(A), J_{+}(A)$, and $J_{-}(A)$ to be well-defined on longer products, we must guarantee that the value obtained using (A.2) and (A.3) does not change if we transform $A$ by any of the relations (2.4) of $\mathrm{S}_{v} \mathrm{U}(2)$ (including the relation $\alpha \gamma=v \gamma \alpha$ ). It suffices to show that if $A=0$ is any of these relations, we get $J(A)=0$, and this is readily verified. In particular, this fixes the relation between the Woronowicz deformation parameter $v$, and the parameter appearing in (A.2).

The three functionals $J \cdot$ are easy to compute directly on any monomial. Let $m, m^{\prime}$ denote monomials in $\alpha$ and $\alpha^{*}$, and let $|m|$ denote the grade of $m$ with respect $\alpha$, i.e. the number of factors $\alpha$ minus the number of factors $\alpha^{*} . \gamma^{\#}$ stands for 
either $\gamma$ or $\gamma^{*}$, and $A \in \mathscr{C}_{0}$ is arbitrary. Then

$$
\begin{aligned}
J_{z}\left(m \gamma^{\sharp} m^{\prime}\right) & =v^{ \pm J_{z}}\left(m \gamma^{\#} m^{\prime}\right)=0, \\
J_{ \pm}\left(m \gamma^{\#} m^{\prime}\right) & =v^{J_{z}}(m) J_{ \pm}\left(\gamma^{\sharp}\right) v^{-J_{z}}\left(m^{\prime}\right), \\
J_{z}(m) & =|m| / 2, \\
v^{ \pm J_{z}}(m) & =v^{ \pm|m| / 2}, \\
J_{ \pm}(m) & =0 \\
J_{z}\left(A^{*}\right) & =-\overline{J_{z}(A)}, \\
J_{+}\left(A^{*}\right) & =-\frac{1}{v} \overline{J_{-}(A)} .
\end{aligned}
$$

Using the definition of the product in $\mathscr{C}_{0}^{*}$ in terms of the coproduct of $\mathscr{C}$, we find the commutation relations

$$
\begin{aligned}
& {\left[J_{z}, J_{ \pm}\right]= \pm J_{ \pm}, \quad J_{ \pm} v^{J_{z}}=v^{\left(J_{z} \mp 1\right)} J_{ \pm},} \\
& {\left[J_{+}, J_{-}\right]=\frac{v}{v^{2}-1}\left(v^{2 J_{z}}-v^{-2 J_{z}}\right) .}
\end{aligned}
$$

Given a unitary representation $u \in \mathscr{M}_{d}(\mathscr{C})$, we can apply the linear functionals $\xi \in \mathscr{C}_{0}^{*}$ to each matrix element, thus obtaining a scalar matrix $\xi(u)$. Then the representation relation $(2.9)$ becomes

$$
(\xi \cdot \eta)(u)=\xi(u) \eta(u)
$$

where on the left we have the product in $\mathscr{C}_{0}^{*}$, and on the right the matrix product. In particular, the commutation relations (A.4) hold in any representation $u$. The unitarity of $u$ becomes a condition on the adjoints of the matrices $J(u)$ :

$$
J_{z}(u)^{*}=J_{z}(u), \quad J_{ \pm}(u)^{*}=J_{\mp}(u) .
$$

The condition of invariance of a state with respect to the action $\alpha_{u}$ can be written directly in terms of the matrices $J(u)$ and the density matrix $\rho$. By applying $J$ to the equation $\sum_{l^{\prime} j^{\prime}} \rho_{l^{\prime} j^{\prime}} u_{j^{\prime} j}\left(u_{i^{\prime} i}\right)^{*}=\rho_{l j}$, and using the unitarity (A.5), we get:

$$
J_{z}(u) \rho=\rho J_{z}(u), \quad J_{+}(u) v^{2 J_{z}(u)} \rho=v^{2 J_{z}(u)} \rho J_{+}(u) .
$$

In particular, $\rho=v^{-2 J_{z}(u)} /[2 S+1]_{v}$ defines an invariant state, and, for an irreducible representation this is the only one.

Acknowledgements. Most of this work was done during visits of M.F. to Osnabrück. He would like to take this opportunity to express his thanks for the warm hospitality and most enjoyable cooperation. R.F.W. was partly supported by a scholarship from the DFG (Bonn). We thank A. Van Daele for pointing out to us the definition of compact quantum group and gratefully acknowledge communications from Y. Watatani, and conversations with A. Yu. Alekseev, H. Grosse, T. Matsui, K.-H. Rehren and P. Vecsernyés. 


\section{References}

[AF] Accardi, L., Frigerio, A.: Markovian Cocycles. Proc. R. Ir. Acad. 83A(2), 251-263 (1983)

[AKLT] Affleck, I., Kennedy, T., Lieb, E.H., Tasaki, H.: Valence bond ground states in isotropic quantum antiferromagnets. Commun. Math. Phys. 115, 477-528 (1988)

[ASW] Alcaraz, F.C., Salinas, S.R., Wreszinski, W.F.: Anisotropic ferromagnetic quantum domains, Phys. Rev. Lett. 75, 930-933(1995)

[BS] Baaj, S., Skandalis, G.: Unitaires multiplicatifs et dualité pour les produits croisés de $C^{*}$-algèbres. Ann. Ec. Norm. Sup. 26, 425 (1993)

[Bab] Babujian, H.M.: Exact solution of the isotropic Heisenberg chain with arbitrary spins: Thermodynamics of the model. Nucl. Phys. B 215, 317-336 (1983)

[BMNR] Batchelor, M.T., Mezincescu, L., Nepomechie, R., Rittenberg, V.: $q$-Deformations of the O(3)-symmetric spin-1 chain. J. Phys. A23, L141-L144 (1990)

[BY] Batchelor, M.T., Yung, C.M.: $q$-Deformations of quantum spin chains with exact valence bond ground states. Preprint archived in cond-mat@babbage.sissa.it \#9403080

[BF] Bernard, D., Felder, G.: Quantum group symmetries in two-dimensional lattice quantum field theory. Nucl. Phys. B 365, 98-120 (1991)

[Ber] Bernard, D.: Quantum group symmetries and non-local currents in 2D QFT. Commun. Math. Phys. 142, 99-138 (1991)

[Bie] Biedenharn, L.C.: A $q$-Boson realization of the quantum group $\mathrm{SU}_{q}(2)$, and the theory of $q$-tensor operators. In: Doebner, H.-D., Hennig, J.-D. (eds.) Quantum groups. Springer Lect. Note: Phys. 370, Berlin, Heidelberg, New York: Springer Verlag, pp. 67-88 1990

[BR] Bratteli, O., Robinson, D.W.: Operator algebras and quantum statistical mechanics. 2 volumes, Berlin, Heidelberg, New York: Springer Verlag, 1979 and 1981

[CE] Choi, M.D., Effros, E.G.: Nuclear $C^{*}$-algebras and the approximation property. Ann. Math. 100, 61-79 (1978)

[Cu1] Cuntz, J.: Simple $C^{*}$-algebras generated by isometries. Commun. Math. Phys. 57, 173185 (1977)

[Cu2] Cuntz, J.: Regular actions of Hopf algebras on the $C^{*}$-algebra generated by a Hilbert space. In: Herman, R., Tanbay, B. (eds.): Operator algebras, mathematical physics, and low dimensional topology. Wellesley, MA: Peters, A.K. 1993

[DC] Dasgupta, N., Chowdhury, A.R.: Algebraic Bethe ansatz with boundary condition for $\mathrm{SU}_{p, q}(2)$ invariant spin chain. J. Phys. A 26 5427-5433 (1993)

[DFJMN] Davies, B., Foda, O., Jimbo, M., Miwa, T., Nakayashiki, A.: Diagonalization of the XXZ Hamiltonian by Vertex Operators. Commun. Math. Phys. 151, 89-153 (1993)

[DHR] Doplicher, S., Haag, R., Roberts, J.E.: Fields, observables and gauge transformations, I. Commun. Math. Phys. 13, 1-23 (1969); Part II in Commun. Math. Phys. 15, 173-200 (1969)

[DR1] Doplicher, S., Roberts, J.E.: Endomorphisms of $C^{*}$-algebras, cross products and duality for compact groups. Ann. Math. 130, 75-119 (1989)

[DR2] Doplicher, S., Roberts, J.E.: $C^{*}$-algebras and duality for compact groups: Why there is a compact group of internal symmetries in particle physics. In: Sénéor, R., Mebkhout, M. (ed.) Proceedings of the International Conference on Mathematical Physics. Singapore: World Scientific, 1986, pp. 489-498

[Dri] Drinfel'd, V.G.: Quantum groups. In: Vol. 1 of the Proceedings of the Int. Congr. Math. Berkeley 1986, New York: Academic Press 1987, pp. 798-820

[DW] Duffield, N.G., Werner, R.F.: Local dynamics of mean-field quantum systems. Helv. Phys. Acta 65, 1016-1054 (1992)

[FNW1] Fannes, M., Nachtergaele, B., Werner, R.F.: Finitely correlated states of quantum spin chains. Commun. Math. Phys. 144, 443-490 (1992)

[FNW2] Fannes, M., Nachtergaele, B., Werner, R.F.: Finitely correlated pure states. J. Funct. Anal. 120, 511-534 (1994)

[FSV] Fannes, M., Spohn, H., Verbeure, A.: Equilibrium states for mean field models. J. Math. Phys. 21, 355-358 (1980)

[GW] Gottstein, C.-T., Werner, R.F.: Ground states of the infinite $q$-deformed Heisenberg ferromagnet. Archived in cond-mat@babbage.sissa.it \#9501123 
[GS] Grosse, H., Raschhofer, E.: Bethe-Ansatz solution of a modified SU(3)-XXZ model. In: Fannes, M., Maes, C., Verbeure, A. (eds.) On three levels; micro-, meso-, and macro-approaches in physics. New York: Plenum Press 1994, pp. 385-392

[Ha1] Haldane, F.D.M.: Exact Jastrow-Gutzwiller resonating-valence-bond ground state of the spin-1/2 antiferromagnetic Heisenberg chain with $1 / r^{2}$ exchange. Phys. Rev. Lett. 60, 635-638 (1988)

[Ha2] Haldane, F.D.M.: Physics of the ideal semion gas: spinons and quantum symmetries of the integrable Haldane-Shastry spin chain. To appear in the Proceedings of the 16th Taniguchi Symposium, Kashikojima, Japan. Okiji, A., Kawakami, N. (eds.) Berlin, Heidelberg, New York: Springer, 1994

[Jim] Jimbo, M.: A $q$-Difference Analogue of $U(g)$ and the Yang-Baxter equation. Lett. Math. Phys. 10, 63-69 (1985)

[JSW] Jørgensen, P.E.T., Schmitt, L.M., Werner, R.F.: Positive representations of general commutation relations allowing Wick ordering. Preprint Osnabrück and Iowa, 1993 archived in funct-an@babbage.sissa.it \#9312004

[KSZ] Klümper, A., Schadschneider, A., Zittartz, J.: Groundstate properties of a generalized VBS-model. Zeits. für Phys. B 87, 281-287 (1992)

[KNW] Konishi, Y., Nagisa, M., Watatani, Y.: Some remarks on actions of compact matrix quantum groups on $C^{*}$-algebras. Pacific. J. Math. 153, 119-128 (1992)

[KS] Kulish, P.P., Sklyanin, E.K.: The general $U_{q}[s l(2)]$ invariant XXZ integrable quantum spin chain. J. Phys. A 24, L435-L439 (1991)

[LB] Lienert, C.R., Butler, P.H.: Racah-Wigner algebra for $q$-deformed algebras. J. Phys. A 25, 1223-1235 (1992)

[MS] Mack, G., Schomerus, V.: Conformal field algebras with quantum symmetry from the theory of superselection sectors. Commun. Math. Phys. 134, 139-196 (1990)

[Maj] Majid, S.: Braided matrix structure of the Sklyanin algebra and of the quantum Lorentz group. Commun. Math. Phys. 156, 607-638 (1993)

[Mat] Wolfram Research, Inc.: Mathematica 2.2, Wolfram Research, Inc., Champaign, Illinois, 1992

[MMP] Meljanac, S., Mileković, M., Pallua, S.: Deformed SU(2) Heisenberg chain. J. Phys. A 24, 581-591 (1991)

[MN] Mezincescu, L., Nepomechie, R.I.: Analytical Bethe Ansatz for quantum-algebrainvariant spin chains. Nucl. Phys. B 372, 597-621 (1992)

[RW] Raggio, G.A., Werner, R.F.: Quantum statistical mechanics of general mean field systems. Helv. Phys. Acta 62, 980-1003 (1989)

[Rue] Ruegg, H.: A simple derivation of the quantum Clebsch-Gordan coefficients for $\mathrm{SU}(2)$. J. Math. Phys. 31, 1085-1087 (1991)

[Sha] Shastri, B.S.: Exact solution of an $S=1 / 2$ Heisenberg antiferromagnetic chain with long-ranged interactions. Phys. Rev. Lett. 60, 639-642 (1988)

[SV] Szlachányi, K., Vecsernyés, P.: Quantum symmetry and braid group statistics in $G$-spin models. Commun. Math. Phys. 156, 127-168 (1993)

[Tak] Takesaki, M.: Theory of operator algebras I. Berlin, Heidelberg, New York: Springer 1979

[Vec] Vecsernyés, P.: On the quantum symmetry of the chiral Ising model. Princeton U. preprint PUPT-1406, archived in hepth@xxx.lanl.gov \#9306118

[Wo1] Woronowicz, S.L.: Twisted SU(2) group. An example of a non-commutative differential calculus. Publ. RIMS, Kyoto 23, 117-181 (1987)

[Wo2] Woronowicz, S.L.: Compact matrix pseudogroups. Commun. Math. Phys. 111, 613-665 (1987)

[Wo3] Woronowicz, S.L.: Differential calculus on compact matrix pseudogroups (quantum groups). Commun. Math. Phys. 122, 125-170 (1989)

[Wo4] Woronowicz, S.L.: Compact quantum groups. In preparation 
